\title{
NON-HOMOGENEOUS BEATTY SEQUENCES LEADING TO INVARIANT GAMES
}

\author{
JULIEN CASSAIGNE, ERIC DUCHÊNE, AND MICHEL RIGO
}

\begin{abstract}
We characterize pairs of complementary non-homogeneous Beatty sequences $\left(A_{n}\right)_{n>0}$ and $\left(B_{n}\right)_{n>0}$, with the restriction $A_{1}=1$ and $B_{1} \geq 3$, for which there exists an invariant take-away game having $\left\{\left(A_{n}, B_{n}\right),\left(B_{n}, A_{n}\right) \mid\right.$ $n>0\} \cup\{(0,0)\}$ as set of $P$-positions. Using the notion of Sturmian word arising in combinatorics on words, this characterization can be translated into a decision procedure relying only on a few algebraic tests about algebraicity or rational independence. This work partially answers to a question of Larsson et al. raised in [1].
\end{abstract}

\section{INTRODUCTION}

A pair of non-homogeneous Beatty sequences $\left(A_{n}\right)_{n>0}$ and $\left(B_{n}\right)_{n>0}$ is given by two positive irrational numbers $\alpha, \beta$ (in this paper, we do not consider any rational modulus) and two real numbers $\gamma, \delta$ such that, for all $n>0$,

$$
A_{n}:=\lfloor n \alpha+\gamma\rfloor, \quad B_{n}:=\lfloor n \beta+\delta\rfloor .
$$

Two sequences are said to be complementary, if

$$
\left\{A_{n} \mid n>0\right\} \text { and }\left\{B_{n} \mid n>0\right\} \text { make a partition of } \mathbb{N}_{>0} \text {. }
$$

The general question addressed in this paper is the following one.

Question 1. Let $\left(A_{n}\right)_{n>0}$ and $\left(B_{n}\right)_{n>0}$ be a pair of complementary non-homogeneous Beatty sequences. Consider the set

$$
\mathcal{P}=\{(0,0)\} \cup\left\{\left(A_{n}, B_{n}\right),\left(B_{n}, A_{n}\right) \mid n>0\right\} .
$$

For convenience, we set $A_{0}=B_{0}=0$. Given any pair $(x, y) \notin \mathcal{P}$ does there exist a pair $(u, v)$ of non-negative integers not in the set

$$
\mathcal{P}-\mathcal{P}=\left\{\left(A_{n}-A_{m}, B_{n}-B_{m}\right),\left(A_{n}-B_{m}, B_{n}-A_{m}\right) \mid m, n \geq 0\right\}
$$

and such that $(x-u, y-v)$ belongs to $\mathcal{P}$.

This problem was originally proposed by Larsson et al. in 11] where a similar question is solved for a large class of sequences including the case of homogeneous Beatty sequences $(\gamma=\delta=0)$. More about the background in given in Section 2.3. As explained in the next subsection, our motivation for this problem stems from combinatorial game theory. It permits to determine the existence of rulesets of particular interest.

Key words and phrases. Two-player combinatorial game, Beatty sequence, Sturmian word, Invariant game, Superadditivity. 
1.1. General vector subtraction games. In [10, Golomb introduced the notion of general vector subtraction games. Given $t$ piles of tokens, a position of such a game is a $t$-tuple of non-negative integers, corresponding to the number of tokens in each pile. A move is also a $t$-tuple of non-negative integers corresponding to the number of tokens that are removed from each pile. Let $p=\left(p_{1}, \ldots, p_{t}\right)$ be a position and $m=\left(m_{1}, \ldots, m_{t}\right)$ be a non-zero move. The move $m$ can be applied to the position $p$ provided that $m \leq p$, i.e., for all $i, m_{i} \leq p_{i}$. The position resulting of the application of $m$ is the $t$-tuple $p-m$. Given a set $\mathcal{M}$ of allowed moves and a starting position $p$, two players alternately apply a move from $\mathcal{M}$. The first player unable to move loses the game. Some instances of vector subtraction game correspond to well-known games found in the literature, such as Nim [3] or Wythoff's game 18 .

A position of a game is said to be a $P$-position (resp., an $N$-position), if there exists a strategy for the second (resp., first) player to win the game, whatever the moves of the other player are. One of the first results in combinatorial game theory (CGT) claims that any move from a $P$-position leads to an $N$-position, and from any $N$-position, there exists a move leading to a $P$-position. Games having different sets of moves may share the same set of $P$-positions. Note that these notions of $P$ and $N$-positions are sufficient to understand the CGT content of this manuscript because we focus essentially on sequences.

In [5], the last two authors introduced the notion of an invariant game in a general context. Roughly speaking, a game is said to be invariant if all moves are "playable from any position", i.e., each allowed move is independent from the position it is played from. In particular, they defined it formally for take-away games, and it turns out that invariant take-away games exactly correspond to the general vector subtraction games of Golomb. If $m=\left(m_{1}, \ldots, m_{t}\right)$ is a non-zero move, then it can be applied to every position $p=\left(p_{1}, \ldots, p_{t}\right)$ such that $p \geq m$.

In that framework, a general question is the following one: given a set $\mathcal{P}$ of $t$ tuples of non-negative integers, does there exist an invariant take-away game whose set of $P$-positions is $\mathcal{P}$ ?

Definition 2. A set $\mathcal{P} \subseteq \mathbb{N}^{t}$ is said admissible if there exists an invariant take-away game whose set of $P$-positions is $\mathcal{P}$.

The link with Question 1 addressed at the begin of this paper is given by the next remark.

Remark 3. To determine if $\mathcal{P}$ is an admissible set, it suffices to check whether the game having the maximal subtraction set $\mathcal{M}=\mathbb{N}^{t} \backslash(\mathcal{P}-\mathcal{P})$ as set of moves admits $\mathcal{P}$ as set of $P$-positions. Indeed, since there must be no move between any two $P$-positions, all the moves inside $\mathcal{P}-\mathcal{P}$ must be forbidden. And if a game having a ruleset $X$ which is a subset of $\mathbb{N}^{t} \backslash(\mathcal{P}-\mathcal{P})$, admits $\mathcal{P}$ as set of $P$-positions, then adding to $X$ moves which are not of the form $\mathcal{P}-\mathcal{P}$ does not change the status (i.e., $P$ - or $N_{-}$) of the positions of the game. Notice that with such a maximal subtraction set $\mathcal{M}$, the classical part where one needs to prove that there is no move from a $P$-position to another $P$-position completely disappears. 
In this context, Question 1 can be restated as deciding whether a given pair of complementary non-homogeneous Beatty sequences is admissible or not.

In this paper, we will consider the case $t=2$ and the set $\mathcal{P}$ is built from pairs of non-negative integers obtained from Beatty sequences as in (2). Even though the homogeneous case was solved in [11] (in fact, much more is proven in that paper), we pursue this introduction with the homogeneous case. We present an alternative (short) proof to let the reader build some intuition on a simpler situation.

1.2. Building some intuition from homogeneous Beatty sequences. In the setting of take-away games, it is usual to have $A_{0}=B_{0}=0$ since $(0,0)$ is a terminal $P$-position. In the homogeneous case, we simply let the indices start with 0 .

Definition 4. Let $\alpha$ be a positive irrational number. A a sequence of integers of the form $(\lfloor n \alpha\rfloor)_{n \geq 0}$ is an homogeneous Beatty sequence. Two sequences $\left(A_{n}\right)_{n \geq 0}$ and $\left(B_{n}\right)_{n \geq 0}$ are complementary if (10) holds.

Theorem 5 (Beatty's theorem). Let $\alpha, \beta>0$ be two irrational numbers. The sequences $(\lfloor n \alpha\rfloor)_{n \geq 0}$ and $(\lfloor n \beta\rfloor)_{n \geq 0}$ are complementary if and only if

$$
\frac{1}{\alpha}+\frac{1}{\beta}=1 \text {. }
$$

The color code (red, green and blue cells) defined in the next example will be standard for the whole paper.

Example 6. Take $\alpha=(1+\sqrt{5}) / 2$ be the golden ratio and $\beta=(3+\sqrt{5}) / 2$ satisfying Beatty's theorem. These two numbers define a pair of complementary homogeneous Beatty sequences $\left(A_{n}\right)_{n \geq 0}=(\lfloor n \alpha\rfloor)_{n \geq 0}$ and $\left(B_{n}\right)_{n \geq 0}=(\lfloor n \beta\rfloor)_{n \geq 0}$. In Figure 1] the set of pairs in $\mathcal{P}=\left\{\left(A_{n}, B_{n}\right),\left(B_{n}, A_{n}\right) \mid n \geq 0\right\}$ is represented in red. It is well-known that $\mathcal{P}$ is the set of $P$-positions of the Wythoff's game [18.

Since $(0,0)$ belongs to $\mathcal{P}$, we have that $\mathcal{P} \subset(\mathcal{P}-\mathcal{P})$. As in Remark 3 , consider a take-away game with the maximal set of moves $\mathcal{M}=\mathbb{N}^{2} \backslash(\mathcal{P}-\mathcal{P})$. The elements in $\mathbb{N}^{2} \cap(\mathcal{P}-\mathcal{P})$ not in $\mathcal{P}$ are represented in blue or green and we will explain the difference below.

Let $(x, y) \notin \mathcal{P}$. There are three cases.

(a) The pair $(x, y)$ corresponds to a white cell if and only if $(x, y) \notin \mathcal{P}-\mathcal{P}$. Hence $(x, y)$ admits a move leading to $(0,0)$. With a take-away game with $\mathcal{M}$ as set of moves, from position $(x, y)$, we can remove all the token in a single move.

(b) If $(x, y)$ corresponds to a green cell, then there exist $(p, q) \in \mathcal{P}$ such that $x>p$ and $y=q$, or $x=p$ and $y>q$. In the first (resp. second) case, $(x-p, 0)($ resp. $(0, y-q))$ is a move leading to $(p, q)$. As a take-away game, this corresponds to the moves of the game of Nim. Since we are dealing with complementary sequences, these moves are always in $\mathcal{M}$. Stated otherwise, for all $m, n$, if $m \neq n$, then $A_{n} \neq A_{m}, B_{m}$ and $B_{n} \neq A_{m}, B_{m}$ or, equivalently the four integers $A_{n}-A_{m}, B_{n}-B_{m}, A_{n}-B_{m}, B_{n}-A_{m}$ are all non-zero. No element in $\mathcal{P}-\mathcal{P}$ is horizontal or vertical.

(c) If $(x, y)$ corresponds to a blue cell, then $(x, y)$ belongs to $\mathcal{P}-\mathcal{P}$ and there is no Nim-move leading to $\mathcal{P}$. These are the only possibly problematic positions. Question 1 therefore becomes in this context: does there exist $(u, v) \in \mathcal{M}$ such that $(x-u, y-v)$ is in $\mathcal{P}$ ? 


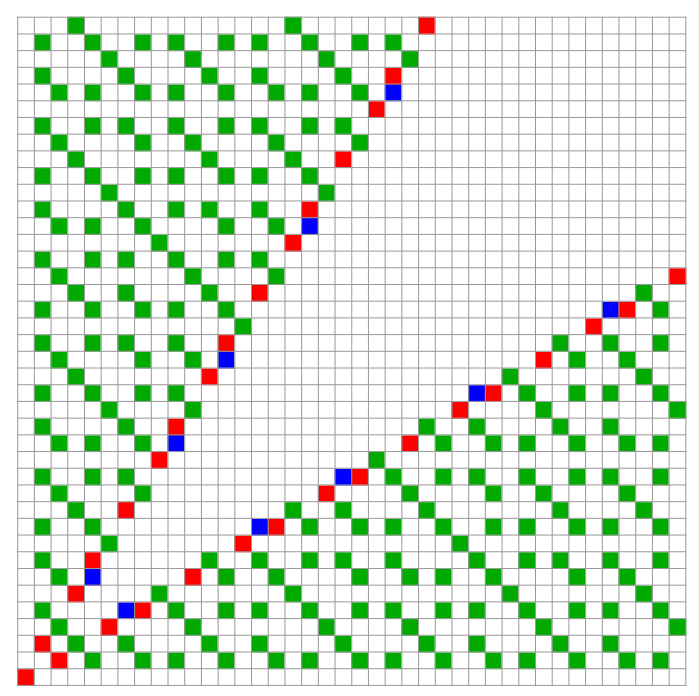

Figure 1 . The set $\mathbb{N}^{2} \cap(\mathcal{P}-\mathcal{P})$ when $\alpha=(1+\sqrt{5}) / 2$.

In Figure 1, we may observe that for every blue cell $(x, y)$, the cell $(x-1, y-1)$ is a red one. Since $(1,1)$ belongs to $\mathcal{M}$, the figure suggests that $\mathcal{P}$ is admissible. (Of course, this is well-known in the case of Wythoff's game.)

Theorem 7. Let $\alpha, \beta$ be irrational numbers with $1<\alpha<2<\beta$ be such that $\left(A_{n}\right)_{n \geq 0}=(\lfloor n \alpha\rfloor)_{n \geq 0}$ and $\left(B_{n}\right)_{n \geq 0}=(\lfloor n \beta\rfloor)_{n \geq 0}$ are complementary homogeneous Beatty sequences. The set $\mathcal{P}=\left\{\left(A_{n}, B_{n}\right),\left(B_{n}, A_{n}\right) \mid n \geq 0\right\}$ is admissible.

Let $\mathcal{M}=\mathbb{N}^{2} \backslash(\mathcal{P}-\mathcal{P})$. We have to prove that for every $(x, y) \notin \mathcal{P}$, there exists $(u, v) \in \mathcal{M}$ such that $(x-u, y-v) \in \mathcal{P}$. It essentially follows from the next three lemmas.

Lemma 8. Let $n>m$ and $\mu$ be a positive irrational number. We have $\lfloor n \mu\rfloor-$ $\lfloor m \mu\rfloor \in\{\lfloor(n-m) \mu\rfloor,\lfloor(n-m) \mu\rfloor+1\}$.

Proof. Observe that

$$
n \mu-2=m \mu+(n-m) \mu-2<\lfloor m \mu\rfloor+\lfloor(n-m) \mu\rfloor \leq m \mu+(n-m) \mu=n \mu .
$$

This yields

$$
\lfloor n \mu\rfloor-2<\lfloor m \mu\rfloor+\lfloor(n-m) \mu\rfloor \leq\lfloor n \mu\rfloor .
$$

The next statement explains the configuration seen in Figure 1 with the blue and green cells occurring in the outer region delimited by the cone defined by the red cells.

Lemma 9. The set $\mathbb{N}^{2} \cap(\mathcal{P}-\mathcal{P})$ is included in

$$
\begin{aligned}
\{0,0\} \cup & \left\{\left(A_{n}+\epsilon-h, B_{n}+\epsilon^{\prime}+h\right),\left(B_{n}+\epsilon^{\prime}+h, A_{n}+\epsilon-h\right)\right. \\
& \left.\mid n \geq 1,0 \leq h<A_{n}, \epsilon, \epsilon^{\prime} \in\{0,1\}\right\} .
\end{aligned}
$$


Proof. Elements of $\mathbb{N}^{2} \cap(\mathcal{P}-\mathcal{P})$ are either $(0,0)$, or $\left(A_{n}-A_{m}, B_{n}-B_{m}\right)$ for $m<n$, or $\left(A_{n}-B_{m}, B_{n}-A_{m}\right)$ for $B_{m}<A_{n}$, or their transpose. By Lemma 8 $\left(A_{n}-A_{m}, B_{n}-B_{m}\right)$ is of the form $\left(A_{n-m}+\epsilon, B_{n-m}+\epsilon^{\prime}\right)$ with $\epsilon, \epsilon^{\prime} \in\{0,1\}$ (i.e., we take $h=0)$. Now, in the second case, by Lemma 8 , there exist $\epsilon, \epsilon^{\prime} \in\{0,1\}$ such that

$$
\begin{aligned}
\left(A_{n}-B_{m}, B_{n}-A_{m}\right) & =\left(A_{n-m}+A_{m}+\epsilon-B_{m}, B_{n-m}+B_{m}+\epsilon^{\prime}-A_{m}\right) \\
& =\left(A_{n-m}+\epsilon-h, B_{n-m}+\epsilon^{\prime}+h\right)
\end{aligned}
$$

with $h=B_{m}-A_{m}>0$ and since $A_{n-m}+\epsilon-h>0$, we conclude that $h \leq A_{n-m}$.

Lemma 10. The elements $(u, 0),(0, v),(1,1)$ belong to $\mathcal{M}$ for all $u, v>0$.

Proof. The fact that $(u, 0)$ and $(0, v)$ belong to $\mathcal{M}$ follows from the complementarity of the sequences, see Example 6 case (b). The fact that $(1,1)$ belongs to $\mathcal{M}$ follows from Lemma 9 because, for all $n \geq 1, h, \epsilon^{\prime} \geq 0, B_{n}+\epsilon^{\prime}+h \geq B_{1} \geq 2$. Hence $(1,1)$ does not belong to $\mathcal{P}-\mathcal{P}$.

Proof of Theorem 7. Without loss of generality, we may assume that $x \leq y$ and $(x, y) \notin \mathcal{P}$. Complementarity implies that $x=A_{i}$ or $x=B_{i}$ for some $i$.

If $x=B_{i}$, then consider the move $(u, v)=\left(0, y-A_{i}\right)$. Since $y \geq B_{i}, y-A_{i}>0$ and $(u, v) \in \mathcal{M}$ by Lemma 10 Hence, $(x, y)-(u, v)=\left(B_{i}, A_{i}\right)$ belongs to $\mathcal{P}$.

If $x=A_{i}$ and $y>B_{i}$, then consider the move $(u, v)=\left(0, y-B_{i}\right)$. We conclude with Lemma 10.

Now, it remains the case $x=A_{i} \leq y<B_{i}$. The case $y=B_{i}$ is obviously excluded as $(x, y) \notin \mathcal{P}$. If $(x, y) \in \mathcal{M}$, then we can consider the move $(u, v)=(x, y)$ leading to $(0,0)$.

Assume that $(x, y) \notin \mathcal{M}$, i.e., $(x, y) \in \mathbb{N}^{2} \backslash(\mathcal{P}-\mathcal{P})$. By Lemma 9, there exist $n \geq 1, \epsilon, \epsilon^{\prime} \in\{0,1\}, h \in\left\{0, \ldots, A_{n}-1\right\}$ such that

$$
x=A_{n}+\epsilon-h \quad \text { and } \quad y=B_{n}+\epsilon^{\prime}+h .
$$

(Note that $x=B_{n}+\epsilon^{\prime}+h, y=A_{n}+\epsilon-h$, is only possible if $x=y=B_{n}+\epsilon^{\prime}+h=$ $A_{n}+\epsilon-h$, since $x \leq y$ and $B_{n}+\epsilon^{\prime}+h \geq B_{n} \geq A_{n}+1 \geq A_{n}+\epsilon-h$, so that the above equalities also hold true.) As $x=A_{i} \leq y<B_{i}$, we have

$$
A_{i}=A_{n}+\epsilon-h \leq A_{n}+1 \leq A_{n+1} \text {, so that } i \leq n+1
$$

and

$$
B_{i}>B_{n}+\epsilon^{\prime}+h \geq B_{n}, \text { so that } i>n .
$$

Hence $i=n+1, \epsilon=1, h=0, x=A_{n+1}=A_{n}+1, y=B_{n}+\epsilon^{\prime}$. So $(x, y)-\left(1, \epsilon^{\prime}\right)=$ $\left(A_{n}, B_{n}\right) \in \mathcal{P}$, with $\left(1, \epsilon^{\prime}\right) \in \mathcal{M}$

1.3. Background on non-homogeneous Beatty sequences. In this paper, we will focus on sets $\mathcal{P}$ of the form (2) built on a pair $\left(A_{n}\right)_{>0}$ and $\left(B_{n}\right)_{n>0}$ of complementary non-homogeneous Beatty sequences defined as follows.

Definition 11. A pair of non-homogeneous complementary Beatty sequences $\left(A_{n}\right)_{n>0}$ and $\left(B_{n}\right)_{n>0}$ is given by two positive irrational numbers $\alpha, \beta$ and two real numbers $\gamma, \delta$ such that, for all $n>0, A_{n}:=\lfloor n \alpha+\gamma\rfloor, B_{n}:=\lfloor n \beta+\delta\rfloor$ and (11) holds 
As noticed in Example 6, given a pair of non-homogeneous complementary Beatty sequences, the Nim moves (i.e., moves of the type $(u, 0)$ and $(0, v))$ belong to $\mathcal{M}=\mathbb{N}^{2} \backslash(\mathcal{P}-\mathcal{P})$, for all $u, v>0$.

In the homogeneous case, we have recalled Beatty's theorem. Similarly, Fraenkel's partition theorem 7] gives necessary and sufficient conditions for complementarity of non-homogeneous Beatty sequences. This result is stated in a more general context (partitions of either $\mathbb{Z}, \mathbb{Z}_{\geq N}$ or $\mathbb{Z}_{\leq N}$ and it deals with irrational $\alpha$ and $\beta$ as well as rational ones). Another resource is [15]. For the sake of clarity, we limit ourselves to a statement of a restricted version of Fraenkel's theorem.

Theorem 12. 7. Thm. II] Let $\alpha, \beta$ be positive irrational numbers. The sequences $(\lfloor n \alpha+\gamma\rfloor)_{n>0}$ and $(\lfloor n \beta+\delta\rfloor)_{n>0}$ are complementary if and only if (3) holds and the following two conditions also hold

$$
\begin{gathered}
\frac{\gamma}{\alpha}+\frac{\delta}{\beta}=\lfloor\alpha+\gamma\rfloor \text { and, } \\
\text { for all } n \geq 1, n \beta+\delta \notin \mathbb{Z} .
\end{gathered}
$$

\section{Considered SETting}

2.1. Our assumptions. Let $\alpha, \beta$ be positive irrational numbers and two real numbers $\gamma, \delta$ such that the two sequences defined, for all $n>0$,

$$
A_{n}:=\lfloor n \alpha+\gamma\rfloor, \quad B_{n}:=\lfloor n \beta+\delta\rfloor
$$

form a pair of complementary non-homogeneous Beatty sequences (thus, From Theorem [12 relations (3), (44), (15) hold). For convenience, we set $A_{0}=B_{0}=0$. Without loss of generality, we assume that $\alpha<\beta$. From Theorem 12 and condition (3), we deduce that $1<\alpha<2<\beta$. In what follows, we moreover assume that

$$
A_{1}=1 \text { and } B_{1} \geq 3 \text {. }
$$

The main purpose of this paper is to answer, in this setting, Question 1, i.e., to characterize the values of $\alpha, \beta, \gamma, \delta$ for which the corresponding set $\mathcal{P}$ defined by (2) is admissible. The condition $B_{1} \geq 3$ is the only limitation of our study, the technical explanation about this extra assumption will come from Lemma 47

Remark 13. The condition $A_{1}=1$ was also considered in the initial problem stated in [11, Problem 2]. In particular, it guarantees that the inequality $A_{n}<B_{n}$ holds for all $n>0$. This condition is not crucial and its removal would not fundamentally change our work. Note that $A_{1}=1$ also implies

$$
1-\alpha \leq \gamma<2-\alpha
$$

Moreover, relation (4) reduces to

$$
\frac{\gamma}{\alpha}+\frac{\delta}{\beta}=1 .
$$


2.2. A few examples. Now that the setting is clear, we present numerical examples suggesting that the admissibility of $\mathcal{P}$ strongly depends on the values of the parameters $\alpha, \beta, \gamma, \delta$ defining the two Beatty sequences. The first two examples are with $\gamma$ negative, the first one gives a non-admissible set, the second one suggests admissibility (the proof of admissibility will follow from our main results, Theorems 45 and 50). The last two examples are with $\gamma$ positive. This distinction about the sign of $\gamma$ will appear all along the paper.

Example 14. Take $\beta=3.99+\sqrt{5} / 2 \simeq 5.108$ and $\gamma=-0.2$. From (3) and (4), we get $\alpha=\beta /(\beta-1) \simeq 1.243$ and $\delta=-\beta \gamma / \alpha \simeq 0.822$. The first elements of $\left(A_{n}, B_{n}\right)_{n>0}$ are

\begin{tabular}{c|cccccccccc}
$n$ & 1 & 2 & 3 & 4 & 5 & 6 & 7 & 8 & 9 & 10 \\
\hline$A_{n}$ & 1 & 2 & 3 & 4 & 6 & 7 & 8 & 9 & 10 & 12 \\
$B_{n}$ & 5 & 11 & 16 & 21 & 26 & 31 & 36 & 41 & 46 & 51
\end{tabular}

The set $\mathbb{N}^{2} \cap(\mathcal{P}-\mathcal{P})$ is represented in Figure 2 with the same color code as in Example6. For the blue cell $(6,2)$, we can remove $(1,1) \notin(\mathcal{P}-\mathcal{P})$ to get $(5,1) \in \mathcal{P}$.

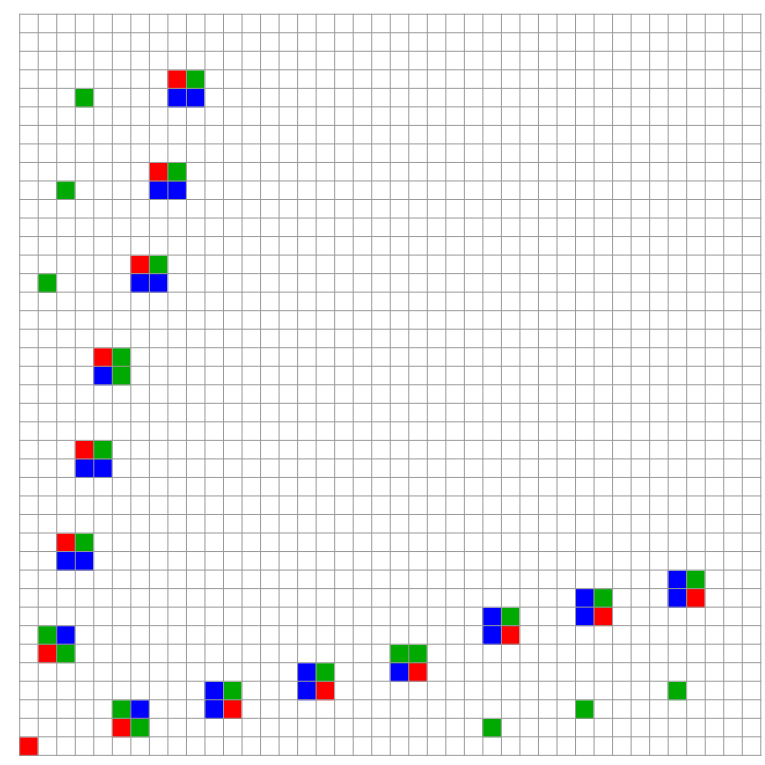

Figure 2. The set $\mathbb{N}^{2} \cap(\mathcal{P}-\mathcal{P})$ with the values of Example 14. $\gamma<0$ and $\mathcal{P}$ is not admissible.

Similarly, with the blue cell $(10,3)$, we can remove $(5,2) \notin(\mathcal{P}-\mathcal{P})$ to again get $(5,1) \in \mathcal{P}$.

Nevertheless, with the blue cell $(10,2)$, the only non-zero element in $\mathcal{P}$ that could possibly be reached is $(5,1)$. But to get that element, we should remove exactly $(5,1)$ which belongs to $\mathcal{P}$. This shows that with these values of $\alpha, \beta, \gamma, \delta$ the corresponding set $\mathcal{P}$ is not admissible. The reader may already notice that $(10,2)$ is of the form $\left(B_{n}+B_{1}, A_{n+1}\right)$ with $n=1$ (this will play a special role in our proofs). 
Example 15. Let us modify slightly the values of the previous example (we subtract 1 to $\beta$ ) and take $\beta=2.99+\sqrt{5} / 2 \simeq 4.108$ and $\gamma=-0.2$. From (3) and (44), we get $\alpha=\beta /(\beta-1) \simeq 1.322$ and $\delta=-\beta \gamma / \alpha \simeq 0.622$. The first elements of $\left(A_{n}, B_{n}\right)_{n>0}$ are

\begin{tabular}{c|cccccccccc}
$n$ & 1 & 2 & 3 & 4 & 5 & 6 & 7 & 8 & 9 & 10 \\
\hline$A_{n}$ & 1 & 2 & 3 & 5 & 6 & 7 & 9 & 10 & 11 & 13 \\
$B_{n}$ & 4 & 8 & 12 & 17 & 21 & 25 & 29 & 33 & 37 & 41
\end{tabular}

The set $\mathbb{N}^{2} \cap(\mathcal{P}-\mathcal{P})$ is represented in Figure 3 with the same color code as in Example 6, For each blue cell $(x, y)$ in Figure 3, we are able to find a pair $(u, v)$

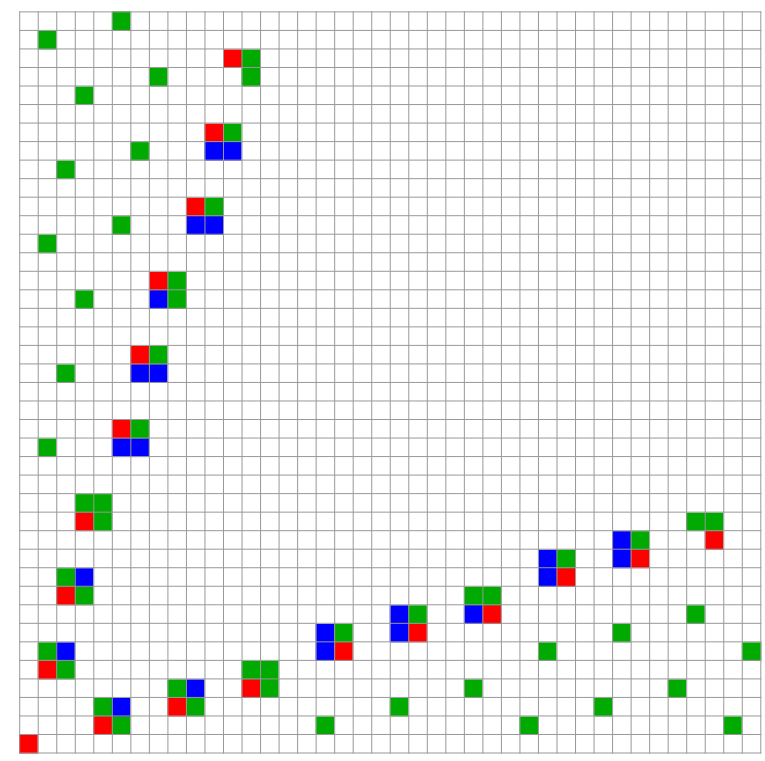

Figure 3. The set $\mathbb{N}^{2} \cap(\mathcal{P}-\mathcal{P})$ with the values of Example 15, $\gamma<0$ and $\mathcal{P}$ should be admissible.

not in $\mathcal{P}-\mathcal{P}$ that answers Question 1] $(x-u, y-v)$ belongs to $\mathcal{P}$, see Table 1 , This example suggests that, with these values of $\alpha, \beta, \gamma, \delta$, the corresponding set $\mathcal{P}$ should be admissible (we have to wait for Theorem 50).

In the first two examples, $\gamma$ was negative. Now, we consider two example where $\gamma$ is positive, the first one gives a non-admissible set, the second one suggests admissibility.

Example 16. Take $\beta=4.99+\sqrt{5} / 2 \simeq 6.108$ and $\delta=-1-\sqrt{2} \simeq-2.414$. From (3i) and (44), we get $\alpha=\beta /(\beta-1) \simeq 1.196$ and $\gamma=-\alpha \delta / \beta \simeq 0.473$. The set $\mathbb{N}^{2} \cap(\mathcal{P}-\mathcal{P})$ is represented in Figure 4 with the same color code as in Example 6 . The blue cell $(6,2)$ belongs to $\mathcal{P}-\mathcal{P}$ and the only non-zero element in $\mathcal{P}$ that can be reached is $(3,1)$ which belongs to $\mathcal{P}$. Hence, with these values of $\alpha, \beta, \gamma, \delta$ the corresponding set $\mathcal{P}$ is not admissible. Again the reader may already notice that $(6,2)$ is of the form $\left(B_{n}+B_{1}, A_{n+1}\right)$ with $n=1$. 


\begin{tabular}{c|c|c}
$(x, y)$ & $\begin{array}{c}(u, v) \\
\notin(\mathcal{P}-\mathcal{P})\end{array}$ & $\begin{array}{c}(x-u, y-v) \\
\in \mathcal{P}\end{array}$ \\
\hline$(5,2)$ & $(1,1)$ & $(4,1)$ \\
$(9,3)$ & $(1,1)$ & $(8,2)$ \\
$(16,5)$ & $(8,3)$ & $(8,2)$ \\
$(16,6)$ & $(8,4)$ & $(8,2)$ \\
$(20,6)$ & $(3,1)$ & $(17,5)$ \\
$(20,7)$ & $(3,2)$ & $(17,5)$ \\
$(24,7)$ & $(3,1)$ & $(21,6)$ \\
$(28,9)$ & $(3,2)$ & $(25,7)$ \\
$(28,10)$ & $(3,3)$ & $(25,7)$ \\
$(32,10)$ & $(3,1)$ & $(29,9)$ \\
$(32,11)$ & $(3,2)$ & $(29,9)$
\end{tabular}

TABLE 1. Blue cells in Example 15.

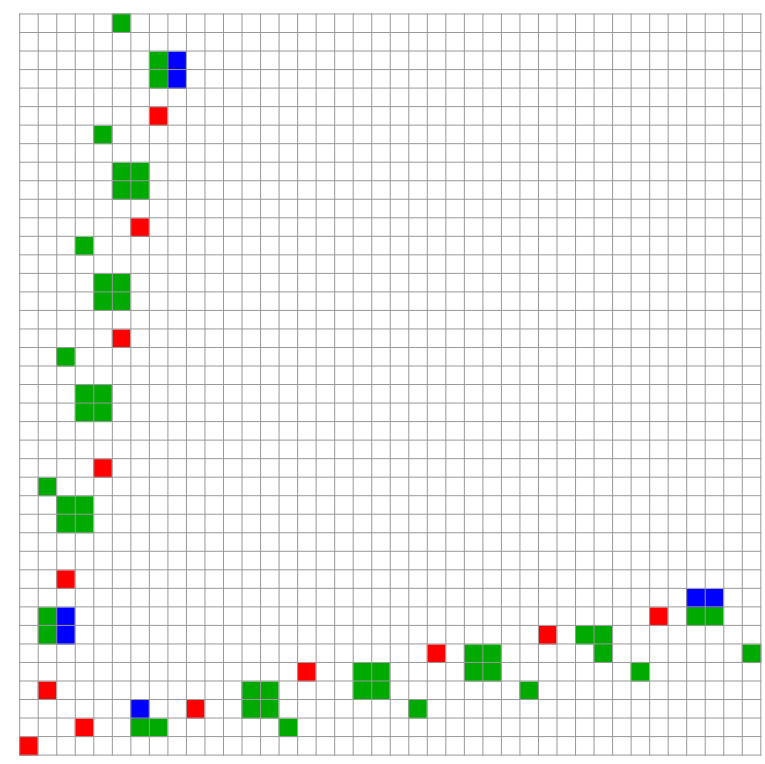

Figure 4. The set $\mathbb{N}^{2} \cap(\mathcal{P}-\mathcal{P})$ with the values of Example 16. $\gamma>0$ and $\mathcal{P}$ is not admissible.

Example 17. Consider the following four real numbers $\beta=8+(1+\sqrt{5}) / 2 \simeq 9.618$, $\alpha=\beta /(\beta-1) \simeq 1.116, \delta=-2.5 \sqrt{7} \simeq-6.614$ and $\gamma=-\delta \alpha / \beta \simeq 0.768$. The set $\mathbb{N}^{2} \cap(\mathcal{P}-\mathcal{P})$ is represented in Figure 5 with the same color code as in Example 6 Figure 5 readily suggests that $\mathcal{P}$ should be admissible. This will be formally proved in Section 7.2. The first terms of the corresponding two sequences are

\begin{tabular}{c|ccccccccccccccc}
$n$ & 1 & 2 & 3 & 4 & 5 & 6 & 7 & 8 & 9 & 10 & 11 & 12 & 13 & 14 & 15 \\
\hline$A_{n}$ & 1 & 2 & 4 & 5 & 6 & 7 & 8 & 9 & 10 & 11 & 13 & 14 & 15 & 16 & 17 \\
$B_{n}$ & 3 & 12 & 22 & 31 & 41 & 51 & 60 & 70 & 79 & 89 & 99 & 108 & 118 & 128 & 137
\end{tabular}




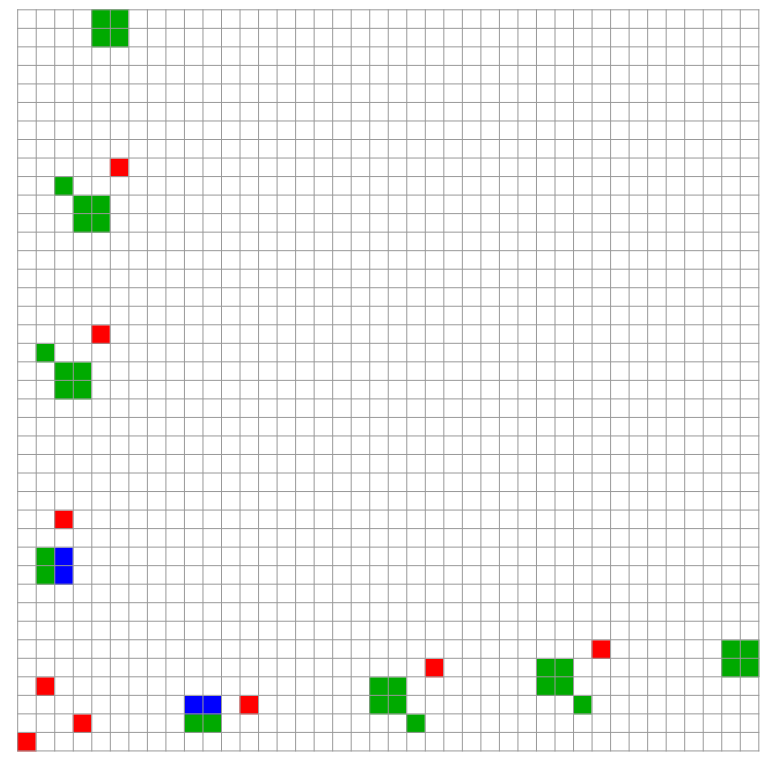

Figure 5. The set $\mathbb{N}^{2} \cap(\mathcal{P}-\mathcal{P})$ with the values of Example 17 , $\gamma>0$ and $\mathcal{P}$ should be admissible.

2.3. Related works. Now that the reader has some intuition about our problem, let us put this paper into perspective. For take-away games played over two piles of token, introducing the notion of an invariant game, admissible sets were first provided in [5] for a particular family of homogeneous Beatty sequences where $\alpha$ is a quadratic irrational number having an ultimately periodic continued fraction expansion of the form $(1 ; \overline{1, k})$. Games related to quadratic irrational numbers with expansion $(1 ; \bar{k})$ were considered in $[8]$. The following conjecture was stated in [5]: any pair $\left(A_{n}, B_{n}\right)_{n \geq 0}$ of complementary (homogeneous) Beatty sequences provides an admissible set.

Later on, Larsson et al. [11] gave sufficient conditions on a pair $\left(A_{n}, B_{n}\right)_{n \geq 0}$ of complementary sequences to provide an admissible set. Their main condition is that the sequence $\left(B_{n}\right)_{n \geq 0}$ is $B_{1}$-superadditive.

Definition 18. A sequence $\left(B_{n}\right)_{n>0}$ is $B_{1}$-superadditive if, for all $m, n>0, B_{m}+$ $B_{n} \leq B_{m+n}<B_{m}+B_{n}+B_{1}$. A sequence satisfying the left-hand inequality is said to be superadditive.

Example 19. The sequence $\left(B_{n}\right)_{n>0}$ of Example 17 is not $B_{1}$-superadditive because we have $B_{1+2}=22>B_{1}+B_{2}+B_{1}$.

The authors of [11] observe that any pair of complementary homogeneous Beatty sequences satisfy in particular this latter property. Therefore, their result provides a positive answer (even for a wider family of complementary sequences) to the conjecture expressed in [5. The problem being solved positively for homogeneous Beatty sequences, it is natural to address Question 1 .

Note that the authors of [1] also wonder whether a pair $\left(A_{n}, B_{n}\right)_{n>0}$ of nonhomogeneous complementary Beatty sequences with $A_{1}=1$ provides an invariant set if and only if the sequence $\left(B_{n}\right)_{>0}$ is $B_{1}$-superadditive. We show in Section 7 
that the answer to this question is negative.

In general, obtaining two games with the same set of $P$-positions is challenging. Recently it was proved that there is no algorithm, given two games played over the same number of heaps (and both described by a finite set of allowed moves), deciding whether or not these two games have the same set of $P$-positions 13 . Thus, taking a maximal subtraction set is a reasonable choice to look for an invariant ruleset.

Let us also mention the introduction of the $\star$-operator in [11. If $G$ is a game (not necessarily invariant), then one can define an invariant game $G^{\star}$ whose set of moves is the set of non-zero $P$-positions of $G$. This dualization operator (see [11. Theorem 1.2]) is a major tool to solve Question 1 for homogeneous Beatty sequences. Let us also mention [12] where the $\star$-operator is used for arbitrary invariant take-away games.

2.4. Organization of the next sections. Our main result will be stated in terms of an infinite word associated with the parameters $\alpha, \beta, \gamma, \delta$, see Definition 34 in Section 4.2. Therefore, in Section 3, we recap the needed background about combinatorics on words and, in particular, about Sturmian words. We are interested in occurrences of some prescribed factor appearing in such a word. Similar interplay between CGT and combinatorics on words can be found in [6, 9].

Section 4 contains the technical core of the paper. When $B_{1} \geq 3$, we provide necessary and sufficient conditions for $\mathcal{P}$ to be an admissible set. Our condition has a combinatorial flavor: we define an infinite word $\mathbf{w}$ built upon $\alpha, \beta, \gamma, \delta$ and the condition is described in terms of factors occurring or not in this word; see Section 4.3. If the 4-tuple $(\alpha, \beta, \gamma, \delta)$ satisfies this condition, then this 4 -tuple is said to satisfy the combinatorial admissibility test or, for short, that the 4-tuple is $C A T$. Finally, in Section 5 , in Theorem 45, we prove that there is no admissible set $\mathcal{P}$ associated with a 4 -tuple which is not CAT. In Theorem 50, we show that if the 4-tuple is CAT, then $\mathcal{P}$ is an admissible set.

In Section 6, we translate these combinatorial conditions into an algebraic setting better suited to tests. It turns out that if $\alpha, \beta, 1$ are rationally independent, then we make use of the two-dimensional version of the density theorem of Kronecker. Otherwise, one has to study the relative position of a rectangle and a straight line over the two-dimensional torus $\mathbb{R}^{2} / \mathbb{Z}^{2}$. This setting is quite natural when studying pairs of natural irrational numbers and can be found in [2, 7, 15, 19.

As already mentioned in Section 2.3] answering a question of Larsson et al. [11, we show in Section 7 that $B_{1}$-superadditivity is not a necessary condition to provide an admissible set.

\section{Sturmian WORDS FOR GAME COMBINATORISTS}

In this section, we collect the main facts on Sturmian words that will be used in this paper. This section has been written for a reader having no particular knowledge in combinatorics on words. For general references, see for instance [1, 14].

Definition 20. An alphabet is a finite non-empty set. Its elements are called symbols. A finite word over the alphabet $\mathcal{A}$ is a finite sequence of elements in $\mathcal{A}$. The set of finite words (resp. finite non-empty words) over $\mathcal{A}$ is denoted by $\mathcal{A}^{*}$ (resp. $\mathcal{A}^{+}$). An infinite word over the alphabet $\mathcal{A}$ is a map from $\mathbb{N}$ to $\mathcal{A}$. Infinite 
words will be denoted using bold face symbols like $\mathbf{s}$ or $\mathbf{w}$. Also note that the first symbol of an infinite word has index 0 .

The so-called Sturmian words form a well-known and extensively studied class of infinite words over a 2-symbol alphabet. They can be defined in several equivalent ways, one of them arising in the context of non-homogeneous Beatty sequences. Below, we present three well-known equivalent definitions of Sturmian words: mechanical words, codings of some rotations and balance property [14, Chap. 2]. We will purposely and interchangeably use these three definitions.

Let $\lambda, \rho$ be two real numbers with $\lambda>0$. In the literature, one usually considers $\lambda \in(0,1)$ which is not a true restriction but, in that case, the obtained word is written over the alphabet $\{0,1\}$. For an arbitrary $\lambda$, the reader will notice that we will have to consider in some situations its fractional part $\{\lambda\}$.

3.1. Mechanical words. We define the infinite word $\mathbf{s}_{\lambda, \rho}=\left(s_{\lambda, \rho}(n)\right)_{n \geq 0}$ by

$$
s_{\lambda, \rho}(n):=\lfloor(n+1) \lambda+\rho\rfloor-\lfloor n \lambda+\rho\rfloor, \quad \forall n \geq 0 .
$$

This word is often referred to as a lower mechanical word [14]. It is not difficult to see that $s_{\lambda, \rho}(n)$ takes exactly the two values $\lfloor\lambda\rfloor$ and $\lfloor\lambda\rfloor+1$.

Example 21. Take $\lambda=\rho=1 / \tau$ where $\tau$ is the golden ratio $(1+\sqrt{5}) / 2$ that we have seen in Example6. The word $\mathbf{s}_{1 / \tau, 1 / \tau}$ is the consecrated Fibonacci word whose first symbols are

$$
\mathbf{s}_{1 / \tau, 1 / \tau}=101101011011010110101 \cdots .
$$

In the context of CGT, this word is a coding of the $P$-positions of Wythoff's games [18, 4.

3.2. Coding of rotations. As we will see, this formalism is convenient to describe the factors occurring in a Sturmian word $\mathbf{s}_{\lambda, \rho}$. Consider the one-dimensional torus $\mathbb{T}^{1}=\mathbb{R} / \mathbb{Z}$ identified with $[0,1)$. Take the map $R_{\lambda}: \mathbb{T}^{1} \rightarrow \mathbb{T}^{1}, x \mapsto\{x+\lambda\}$. One can study the orbit of $\rho$ (reduced modulo 1 ) under the action of $R_{\lambda}$. We thus define two intervals

$$
I_{\lfloor\lambda\rfloor}:=[0,1-\{\lambda\}) \quad \text { and } \quad I_{\lfloor\lambda\rfloor+1}:=[1-\{\lambda\}, 1)
$$

partitioning $\mathbb{T}^{1}$. One can show that this setting provides another way to define the word $\mathbf{s}_{\lambda, \rho}$.

Theorem 22. [14] For all $n \geq 0$, we have

$$
s_{\lambda, \rho}(n)=\lfloor\lambda\rfloor \Leftrightarrow R_{\lambda}^{n}(\rho) \in I_{\lfloor\lambda\rfloor} \text { and } s_{\lambda, \rho}(n)=\lfloor\lambda\rfloor+1 \Leftrightarrow R_{\lambda}^{n}(\rho) \in I_{\lfloor\lambda\rfloor+1} .
$$

Definition 23. A factor of length $\ell>0$ in a word $\mathbf{z}=z_{0} z_{1} \cdots$ is a finite sequence made of $\ell$ consecutive symbols: $z_{i} \cdots z_{i+\ell-1}$. We say that the factor occurs in $\mathbf{z}$ in position $i$. By convention, the unique factor of length 0 is the empty word $\varepsilon$.

Example 24. Considering again the Fibonacci word (7), we see that 011 is a factor of $\mathbf{s}_{1 / \tau, 1 / \tau}$ occurring in positions $1,6,9$.

The set of all factors of length $\ell$ occurring in an infinite word $\mathbf{z}$ is denoted by $\operatorname{Fac}_{\mathbf{z}}(\ell)$ and the whole set of factors of $\mathbf{z}$ is

$$
\operatorname{Fac}_{\mathbf{z}}=\bigcup_{\ell \geq 0} \operatorname{Fac}_{\mathbf{z}}(\ell)
$$


For a binary word $v=v_{0} v_{1} \cdots v_{m}$, for all $t, v_{t} \in\{\lfloor\lambda\rfloor,\lfloor\lambda\rfloor+1\}$, we define a halfinterval $I_{v, \lambda}$ of $\mathbb{T}^{1}$ as

$$
I_{v, \lambda}:=I_{v_{0}} \cap R_{\lambda}^{-1}\left(I_{v_{1}}\right) \cap \cdots \cap R_{\lambda}^{-m}\left(I_{v_{m}}\right) .
$$

One can show that $v$ occurs in $\mathbf{s}_{\lambda, \rho}$ in position $i$ if and only if $R_{\lambda}^{i}(\rho) \in I_{v, \lambda}$. See [14, Section 2.1.2]. This result is illustrated by the following example.

Example 25. Again for the Fibonacci word and the factor 011, recalling that $\tau^{2}-\tau-1=0$, we have

$$
I_{011,1 / \tau}=I_{0} \cap R_{1 / \tau}^{-1}\left(I_{1}\right) \cap R_{1 / \tau}^{-2}\left(I_{1}\right)=\left[0,1 / \tau^{2}\right) \cap \underbrace{R_{1 / \tau}^{-1}\left[1 / \tau^{2}, 1\right)}_{\left[2 / \tau^{2}, 1\right) \cup\left[0,1 / \tau^{2}\right)} \cap \underbrace{R_{1 / \tau}^{-2}\left[1 / \tau^{2}, 1\right)}_{\left[3 / \tau^{2}-1,2 / \tau^{2}\right)}
$$

and we find $I_{011,1 / \tau}=\left[3 / \tau^{2}-1,1 / \tau^{2}\right)$ where $3 / \tau^{2}-1 \simeq 0.146$ and $1 / \tau^{2} \simeq 0.382$. If we compute the first few approximations of $R_{1 / \tau}^{n}(1 / \tau)$ for $n=0,1, \ldots, 10$, we get $0.618,0.236,0.854,0.472,0.0902,0.708,0.326,0.944,0.562,0.180,0.798$. Observe that the only values falling into the interval $I_{011,1 / \tau}$ are for those $n=1,6,9$ which exactly corresponds to the observations about the factor 011 made in Example 24 .

3.3. A balance property. Let $\mathcal{A}$ be a finite alphabet. Let $a$ be a symbol in $\mathcal{A}$ and let $u$ be a finite word over $\mathcal{A}$. We denote by $|u|$ the length of $u$ and by $|u|_{a}$ the number of occurrences of $a$ in $u$.

Definition 26. An infinite word $\mathbf{z}$ over $\mathcal{A}$ is said to be balanced if, for all $n \geq 0$, all $u, v \in \operatorname{Fac}_{\mathbf{z}}(n)$ and all $a \in \mathcal{A}$, we have $\left.|| u\right|_{a}-|v|_{a} \mid \leq 1$.

Theorem 27. [14, Theorem 2.1.5] An infinite word over $\{0,1\}$ is Sturmian if and only if it is aperiodic and balanced.

This result implies that, for a given Sturmian word $\mathbf{s}_{\lambda, \rho}$, up to permutation of the symbols occurring in a factor (i.e., up to abelian equivalence), there are exactly two kinds of factors of length $\ell$, those having either $\lceil\ell\{\lambda\}\rceil$, or $\lceil\ell\{\lambda\}\rceil-1$, symbols $\lfloor\lambda\rfloor+1$. The corresponding factors will be called respectively heavy and light. In [16], the following intervals are defined, for all $\ell>0$,

$$
I_{H, \lambda}(\ell):=[1-\{\ell \lambda\}, 1) \quad \text { and } \quad I_{L, \lambda}(\ell):=[0,1-\{\ell \lambda\})
$$

and it is proved that the factor of length $\ell$ occurring in position $i$ in $\mathbf{s}_{\lambda, \rho}$ is heavy if and only if $R_{\lambda}^{i}(\rho) \in I_{H, \lambda}(\ell)$.

Example 28. For the Fibonacci word introduced in Example 21, there are exactly 6 factors of length 5. Five are light: 10110, 01101, 11010, 10101 and 01011. They contain $\lceil 5\{\tau\}\rceil-1=3$ symbols 1 . The unique heavy factor of length 5 is 11011 with 4 symbols 1 . We have $I_{H, 1 / \tau}(5)=[1-\{5 / \tau\}, 1)$ where $1-\{5 / \tau\} \simeq 0.91$. We have given approximations of the first few values of $R_{1 / \tau}^{n}(1 / \tau)$ in Example 25 and we see that for $n=7$ the point belongs to that interval. Otherwise stated, an heavy factor of length 5 occurs for the first time in position 7 in the Fibonacci word.

We will often make use of the following observation.

Remark 29. Let $u=u_{1} \cdots u_{n}$ and $v=v_{1} \cdots v_{n}$ be two factors of length $n$ occurring in the Sturmian word $\mathbf{s}_{\lambda, \rho}$ over the alphabet $\{\lfloor\lambda\rfloor,\lfloor\lambda\rfloor+1\}$. We have

$$
\sum_{i=1}^{n} u_{i}-\sum_{i=1}^{n} v_{i}=\left\{\begin{aligned}
1 & \text { if } u \text { is heavy and } v \text { is light, } \\
-1 & \text { if } u \text { is light and } v \text { is heavy, } \\
0 & \text { if } u, v \text { are both light (resp., heavy). }
\end{aligned}\right.
$$


In particular, we have

$$
\sum_{i=1}^{n} v_{i}-1 \leq \sum_{i=1}^{n} u_{i} \leq \sum_{i=1}^{n} v_{i}+1 .
$$

\section{A Characterization of the 4-Tuples LEADing to AN INVARiant game}

From $\alpha, \beta, \gamma, \delta$, our first aim is to define an infinite word $\mathbf{w}=\left(w_{n}\right)_{n \geq 0}$ of pairs taking values in a finite set. We proceed with two steps. First, we define the product of two infinite words in Section 4.1. Then we specify our coding word in Section 4.2. We describe some combinatorial conditions on the factors of this coding word in Section 4.3. Finally, in Section 5 we prove that this condition (called CAT) is equivalent to the fact that $\mathcal{P}$ is admissible.

4.1. Direct product and synchronization. In the next section, coming back to our initial problem, we will introduce an infinite word over an alphabet of pairs. This infinite word is obtained as the direct product of two Sturmian words.

Definition 30. Let $\mathbf{s}=\left(s_{n}\right)_{n \geq 0}$ and $\mathbf{t}=\left(t_{n}\right)_{n \geq 0}$ be two infinite words over the alphabets $\mathcal{A}$ and $\mathcal{B}$ respectively. The direct product of $\mathbf{s}$ and $\mathbf{t}$ is the word $\mathbf{s} \otimes \mathbf{t}=$ $\left(p_{n}\right)_{n \geq 0}$ where

$$
p_{n}=\left(s_{n}, t_{n}\right), \quad \forall n \geq 0 .
$$

Observe that $\mathbf{s} \otimes \mathbf{t}$ is an infinite word over the alphabet $\mathcal{A} \times \mathcal{B}$ of pairs of symbols.

We denote by $\pi_{1}$ and $\pi_{2}$ the two homomorphisms of projection defined by $\pi_{1}(x, y)=x, \pi_{2}(x, y)=y$, for all $(x, y) \in \mathcal{A} \times \mathcal{B}$, and extended to $\pi_{1}(\mathbf{s} \otimes \mathbf{t})=\mathbf{s}$ and $\pi_{2}(\mathbf{s} \otimes \mathbf{t})=\mathbf{t}$. If $u \in \mathcal{A}^{+}$and $v \in \mathcal{B}^{+}$are two finite words of the same length, one can define accordingly $u \otimes v$ and $\pi_{1}(u \otimes v)=u, \pi_{2}(u \otimes v)=v$. For more on two-dimensional generalization of Sturmian words, see for instance 2]. For some recurrence properties of direct product, see [17].

Let us now introduce a convenient notation for a particular 2-symbol alphabet denoted by $\mathcal{A}_{\lambda}$. In the next sections, up to some minor modifications of the first symbol, we will be interested in the direct product of two Sturmian words $\mathbf{s}_{\lambda, \rho}$ and $\mathbf{s}_{\mu, \nu}$ over the alphabets $\mathcal{A}_{\lambda}=\{\lfloor\lambda\rfloor,\lfloor\lambda\rfloor+1\}$ and $\mathcal{A}_{\mu}=\{\lfloor\mu\rfloor,\lfloor\mu\rfloor+1\}$ respectively.

Consider the two-dimensional torus $\mathbb{T}^{2}=\mathbb{R}^{2} / \mathbb{Z}^{2}$ identified with $[0,1) \times[0,1)$ and the map $R_{\lambda, \mu}: \mathbb{T}^{2} \rightarrow \mathbb{T}^{2},(x, y) \mapsto(\{x+\lambda\},\{y+\mu\})$. From the characterization given in Section 3.2, it is obvious that $\mathbb{T}^{2}$ is split into four regions of the kind $I_{a} \times I_{b}$ where $a \in \mathcal{A}_{\lambda}$ and $b \in \mathcal{A}_{\mu}$ in such a way that

$$
\left(\mathbf{s}_{\lambda, \rho} \otimes \mathbf{s}_{\mu, \nu}\right)(n)=(a, b) \Leftrightarrow R_{\lambda, \mu}^{n}(\rho, \nu) \in I_{a} \times I_{b} .
$$

Example 31 (Continuing Example 14). Recall that we had $\beta=3.99+\sqrt{5} / 2 \simeq$ 5.108 and $\alpha=\beta /(\beta-1) \simeq 1.243$. We have $\mathcal{A}_{\alpha}=\{1,2\}$ and $\mathcal{A}_{\beta}=\{5,6\}$. In Figure 6, the starting point $(\{\gamma\},\{\delta\})$ is denoted by 0 and the arrow represents the application of $R_{\alpha, \beta}$, i.e., a translation of $(\{\alpha\},\{\beta\})$ in $\mathbb{T}^{2}$. The first ten iterations of this map are labeled in the figure. The torus is split into the four regions corresponding to an occurrence of $(1,5),(1,6),(2,5)$ and $(2,6)$ respectively. These regions are denoted respectively $a, b, c$ and $d$. One can notice that the visited regions are: cbaacaaaacb $\cdots$. From this, we get $\pi_{1}\left(\mathbf{s}_{\alpha, \gamma}\right)=21112111121 \cdots$ and $\pi_{2}\left(\mathbf{s}_{\beta, \delta}\right)=565555555556 \cdots$.

The next statement is a direct reformulation of (8). 


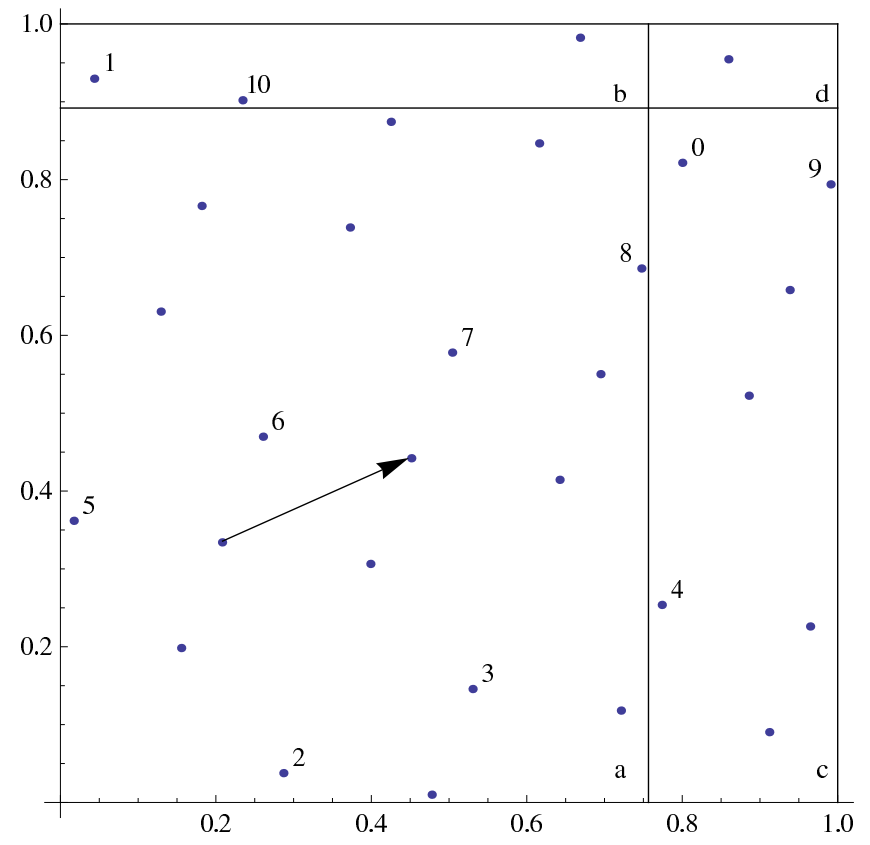

FIgURE 6. The torus $\mathbb{T}^{2}$ split into four regions as discussed in Example 31

Lemma 32. Let $v \in \mathcal{A}_{\lambda}^{+}$and $w \in \mathcal{A}_{\mu}^{+}$be two words of the same length. The factor $v \otimes w$ occurs in $\mathbf{s}_{\lambda, \rho} \otimes \mathbf{s}_{\mu, \nu}$ in position $i$, i.e., $v$ occurs in $\mathbf{s}_{\lambda, \rho}$ in position $i$ and simultaneously $w$ occurs in $\mathbf{s}_{\mu, \nu}$ in position $i$, if and only if $R_{\lambda, \mu}^{i}(\rho, \nu) \in I_{v, \lambda} \times I_{w, \mu}$.

Let $\ell \geq 1$. Using (9), again $\mathbb{T}^{2}$ is split into four regions of the kind $I_{A, \lambda}(\ell) \times I_{B, \mu}(\ell)$ where $A, B \in\{L, H\}$ in such a way that the factor of length $\ell$ occurring in $\mathbf{s}_{\lambda, \rho}$ is light and, simultaneously, the factor of length $\ell$ occurring in $\mathbf{s}_{\mu, \nu}$ is light, if and only if $R_{\lambda, \mu}^{i}(\rho, \nu) \in I_{L, \lambda}(\ell) \times I_{L, \mu}(\ell)$. The other combinations light/heavy, heavy/light and heavy/heavy are obtained accordingly.

4.2. Introducing a coding word. From $\alpha, \beta, \gamma, \delta$, let us define an infinite word $\mathbf{w}=\left(w_{n}\right)_{n \geq 0}$ of pairs taking values in a finite set. Except maybe for the first symbol, this word is the direct product $\mathbf{s}_{\alpha, \gamma} \otimes \mathbf{s}_{\beta, \delta}$ of two Sturmian words. Indeed, we have set $A_{0}=0$ (resp., $B_{0}=0$ ) which may differ from $\lfloor\gamma\rfloor$ (resp., $\lfloor\delta\rfloor$ ).

Let us first collect a few useful relations.

Remark 33. Since we consider complementary sequences, condition (4) in Theorem 12 and our first assumptions on $\alpha$ and $\beta$ (see Section 2.1) imply the following dependencies:

$$
\begin{gathered}
\text { If } 0 \leq \gamma<2-\alpha \text {, then we have } \delta \leq 0 . \\
\text { If } 1-\alpha<\gamma<0 \text {, then we get } 0<\delta<1 .
\end{gathered}
$$

Indeed, (10) is directly deduced from Theorem 12 and (4) because $\delta=-\gamma \beta / \alpha$ and thus $\gamma \delta \leq 0$. For the same reason, in (11), if $\gamma$ is negative, then $\delta$ is positive. 
Moreover, since $\delta=-\gamma \beta / \alpha$ and if we assume that $-\gamma<\alpha-1$, then using (3) we get $\delta<\frac{\beta(\alpha-1)}{\alpha}=1$.

Definition 34 (Introduction of the word $\mathbf{w}$ ). For all $n \geq 0$, we set

$$
w_{n}=\left(A_{n+1}-A_{n}, B_{n+1}-B_{n}\right),
$$

that is

$$
\mathbf{w}=w_{0} w_{1} w_{2} \cdots=\left(A_{1}, B_{1}\right)\left(s_{\alpha, \gamma}(1), s_{\beta, \delta}(1)\right)\left(s_{\alpha, \gamma}(2), s_{\beta, \delta}(2)\right) \cdots
$$

The purpose of this word $\mathbf{w}$ is the following one. Let $i \leq j$. Observe that

$$
\sum_{k=i}^{j} w_{i}=\left(A_{j+1}-A_{i}, B_{j+1}-B_{i}\right) .
$$

Referring to the sign of $\gamma$, this word will be denoted by $\mathbf{w}_{+}$(resp., $\mathbf{w}_{-}$) whenever $\gamma$ is positive (resp., negative). Since $1<\alpha<2$, we have that for all $n \geq 0$, $s_{\alpha, \gamma}(n) \in\{1,2\}$. We also have $s_{\beta, \delta}(n) \in\{\lfloor\beta\rfloor,\lfloor\beta\rfloor+1\}$. Since $A_{1}-A_{0}=1$, the following observation is straightforward.

Lemma 35. For all $n \geq 0$, we have $A_{n+1}-A_{n} \in\{1,2\}$. For all $n>0$, we have $B_{n+1}-B_{n} \in\{\lfloor\beta\rfloor,\lfloor\beta\rfloor+1\}$

Assume first that $\gamma<0$. Hence $\lfloor\gamma\rfloor \leq-1$ and from (11), $0<\delta<1$, i.e., $\lfloor\delta\rfloor=0$. Then $A_{1}<s_{\alpha, \gamma}(0)=A_{1}-\lfloor\gamma\rfloor$ and $B_{1}=B_{1}-\lfloor\delta\rfloor=s_{\beta, \delta}(0)$, i.e., $\pi_{2}\left(\mathbf{w}_{-}\right)=\mathbf{s}_{\beta, \delta}$. The word $\mathbf{w}_{-}$is thus written over the alphabet defined in the next definition.

Definition 36 (Introduction of the 4-symbol alphabet $\{a, b, c, d\}$ ). The word $\mathbf{w}_{-}$ is written over the alphabet of size 4

$$
\{(1,\lfloor\beta\rfloor),(1,\lfloor\beta\rfloor+1),(2,\lfloor\beta\rfloor),(2,\lfloor\beta\rfloor+1)\} .
$$

This set is in one-to-one correspondence with the alphabet $\{a, b, c, d\}$. We choose $a, b, c, d$ such that $\pi_{1}(a)=\pi_{1}(b)=1, \pi_{1}(c)=\pi_{1}(d)=2, \pi_{2}(a)=\pi_{2}(c)=\lfloor\beta\rfloor$ and $\pi_{2}(b)=\pi_{2}(d)=\lfloor\beta\rfloor+1$.

Assume now that $\gamma>0$. Let us explain why we need to introduce a fifth symbol in the alphabet (contrarily to Definition 36). In our setting, recall that $\gamma<1$. Hence $\lfloor\gamma\rfloor=0$ and we get that $A_{1}=s_{\alpha, \gamma}(0)$, i.e., $\pi_{1}\left(\mathbf{w}_{+}\right)=\mathbf{s}_{\alpha, \gamma}$. Moreover, $\delta<0$ and therefore $B_{1}=\lfloor\beta+\delta\rfloor<s_{\beta, \delta}(0)=\lfloor\beta+\delta\rfloor-\lfloor\delta\rfloor$. The word $w_{1} w_{2} \cdots$ is thus written over the alphabet $\{(1,\lfloor\beta\rfloor),(1,\lfloor\beta\rfloor+1),(2,\lfloor\beta\rfloor),(2,\lfloor\beta\rfloor+1)\}$ and $w_{0}=\left(1, B_{1}\right)$ with $B_{1} \leq\lfloor\beta\rfloor$. If $B_{1}<\lfloor\beta\rfloor$, then $\mathbf{w}_{+}$is written over the 5 -symbol alphabet

$$
\left\{\left(1, B_{1}\right),(1,\lfloor\beta\rfloor),(1,\lfloor\beta\rfloor+1),(2,\lfloor\beta\rfloor),(2,\lfloor\beta\rfloor+1)\right\}
$$

the symbol $\left(1, B_{1}\right)$ appearing only once as the first element.

Definition 37 (Introduction of the fifth symbol $e$ ). We set $e$ such that $\pi_{1}(e)=1$ and $\pi_{2}(e)=B_{1}$.

Example 38 (For $\gamma<0$ ). Take the same values as in Examples 14 and 31 leading to a non-admissible set $\mathcal{P}$ (see Figure 21). We get the following table.

\begin{tabular}{r|ccccccccccccccccccc} 
& 0 & 1 & 2 & 3 & 4 & 5 & 6 & 7 & 8 & 9 & 10 & 11 & 12 & 13 & 14 & 15 & 16 & 17 & 18 \\
\hline$A_{n+1}-A_{n}$ & 1 & 1 & 1 & 1 & 2 & 1 & 1 & 1 & 1 & 2 & 1 & 1 & 1 & 2 & 1 & 1 & 1 & 2 & 1 \\
$B_{n+1}-B_{n}$ & 5 & 6 & 5 & 5 & 5 & 5 & 5 & 5 & 5 & 5 & 6 & 5 & 5 & 5 & 5 & 5 & 5 & 5 & 5 \\
\hline $\mathbf{w}_{-}$ & $a$ & $b$ & $a$ & $a$ & $c$ & $a$ & $a$ & $a$ & $a$ & $c$ & $b$ & $a$ & $a$ & $c$ & $a$ & $a$ & $a$ & $c$ & $a$
\end{tabular}


Note that $s_{\alpha, \gamma}(0)=2 \neq 1$ meaning that the first sequence in the above table disagrees with $\mathbf{s}_{\alpha, \gamma}$ for the first term only. Also, the first occurrence of $(2,6)=d$ is for $n=30$. Again, $\mathbf{w}_{-}$agrees with the coding of the trajectory described in Example 31, except for the first symbol.

Example 39 (For $\gamma>0$, continuing Example 16). Recall that we had $\beta=4.99+$ $\sqrt{5} / 2 \simeq 6.108$ and $\alpha=\beta /(\beta-1) \simeq 1.196$ leading to a non-admissible set $\mathcal{P}$ (see Figure 4).

\begin{tabular}{r|ccccccccccccccccccc} 
& 0 & 1 & 2 & 3 & 4 & 5 & 6 & 7 & 8 & 9 & 10 & 11 & 12 & 13 & 14 & 15 & 16 & 17 & 18 \\
\hline$A_{n+1}-A_{n}$ & 1 & 1 & 2 & 1 & 1 & 1 & 1 & 2 & 1 & 1 & 1 & 1 & 2 & 1 & 1 & 1 & 1 & 1 & 2 \\
$B_{n+1}-B_{n}$ & 3 & 6 & 6 & 7 & 6 & 6 & 6 & 6 & 6 & 6 & 6 & 6 & 6 & 7 & 6 & 6 & 6 & 6 & 6 \\
\hline $\mathbf{w}_{+}$ & $e$ & $a$ & $c$ & $b$ & $a$ & $a$ & $a$ & $c$ & $a$ & $a$ & $a$ & $a$ & $c$ & $b$ & $a$ & $a$ & $a$ & $a$ & $c$
\end{tabular}

Note that $s_{\beta, \delta}(0)=6 \neq 3$ meaning that the second sequence in the above table disagrees with $\mathbf{s}_{\beta, \delta}$ for the first term only. Also, the first occurrence of $(2,7)=d$ is for $n=162$.

Example 40 (For $\gamma>0$ ). Take $\beta=4.99+\sqrt{5} / 2 \simeq 6.108$ and $\delta=-0.05$. Hence $B_{1}-B_{0}=\lfloor\beta+\delta\rfloor=6$. Even if $B_{1}-B_{0}$ belong to $\{\lfloor\beta\rfloor,\lfloor\beta\rfloor+1\}$, this example shows that $B_{1}-B_{0} \neq s_{\beta, \delta}(0)=\lfloor\beta+\delta\rfloor-\lfloor\delta\rfloor=7$.

4.3. The combinatorial admissibility condition. It is now convenient to introduce the Parikh vector of a finite word $w \in\{a, b, c, d\}$. It is defined as

$$
\Psi(w)=\left(|w|_{a},|w|_{b},|w|_{c},|w|_{d}\right) \in \mathbb{N}^{4} .
$$

Our aim is to introduce a test on words and factors. In the theory of complexity, the notion of succinct certificates of disqualification is well-known for $c o-N P$ languages. Here we introduce two definitions (depending on the sign of $\gamma$ ) to carry a similar idea. If we find a prefix or a factor with some prescribed properties, then we will show that such an occurrence leads to a non-admissible set $\mathcal{P}$. Roughly speaking, such a prefix or factor is a succinct certificate for non-admissibility.

The following two definitions correspond to the determination of disqualifying factors in $\mathbf{w}_{+}$(for Definition 41) and $\mathbf{w}_{-}$(for Definition 42) respectively.

Definition 41. Let $p=w_{0} u_{1} \cdots u_{n}=w_{0} u$ be the prefix of length $n+1$ of $\mathbf{w}_{+}$. We say that $p$ is disqualifying ${ }^{+}$if $u \in\{a, b\}^{*}, u_{1} \cdots u_{n-1}$ is a palindrome and if any of the following three situations occur:

(i) $2 B_{1}=\pi_{2}\left(u_{n}\right)-1$ and there exists $v \in \operatorname{Fac}_{n}\left(\mathbf{w}_{+}\right)$such that

$$
\Psi(v)=\Psi(u)+(0,-1,1,0) \text { or } \Psi(v)=\Psi(u)+(1,-2,0,1)
$$

(ii) $2 B_{1}=\pi_{2}\left(u_{n}\right)$ and there exists $v \in \operatorname{Fac}_{n}\left(\mathbf{w}_{+}\right)$such that

$$
\Psi(v)=\Psi(u)+(-1,0,1,0) \text { or } \Psi(v)=\Psi(u)+(0,-1,0,1)
$$

(iii) $2 B_{1}=\pi_{2}\left(u_{n}\right)+1$ and there exists $v \in \operatorname{Fac}_{n}\left(\mathbf{w}_{+}\right)$such that

$$
\Psi(v)=\Psi(u)+(-1,0,0,1) \text { or } \Psi(v)=\Psi(u)+(-2,1,1,0)
$$

Although Definition 41 seems rather artificial, its motivation comes from summing up the symbols occurring in such factors. Let $p=w_{0} u_{1} \cdots u_{n}=w_{0} u$ be the prefix of length $n+1$ of $\mathbf{w}_{+}$. First note that

$$
\sum_{i=1}^{n} u_{i}=\left(A_{n}-A_{1}+\pi_{1}\left(u_{n}\right), B_{n}-B_{1}+\pi_{2}\left(u_{n}\right)\right)
$$


The existence of a factor $v \in \operatorname{Fac}_{n}\left(\mathbf{w}_{+}\right)$in one of the three situations means that

$$
\sum_{i=1}^{n} v_{i}=\left(A_{n}+\pi_{1}\left(u_{n}\right), B_{n}+B_{1}\right)=\left(A_{n+1}, B_{n}+B_{1}\right)
$$

We will see later on that such factors prevent us to find a winning strategy from some $N$-positions obtained from $\left(A_{n+1}, B_{n}+B_{1}\right)$.

Definition 42. Let $u=u_{1} \cdots u_{n} \in\{a, b\}^{*}$ be a factor of length $n$ occurring in $\mathbf{w}_{-}$.

(SD.1) If there exists $v \in \operatorname{Fac}_{n}\left(\mathbf{w}_{-}\right) \cap\{a, b\}^{*}$ such that $|v|_{b}<|u|_{b}$, i.e.,

$$
\Psi(v)=\Psi(u)+(j,-j, 0,0), \text { for some } j>0,
$$

then we say that $u$ satisfies property (SD.1) (SD stands for "suffix-disqualifying" as described next).

(SD.2) If there exists $v \in \operatorname{Fac}_{n-1}\left(\mathbf{w}_{-}\right)$such that either $\left|u_{1} \cdots u_{n-1}\right|_{a}=|v|_{a}$, $\left|u_{1} \cdots u_{n-1}\right|_{b}-1=|v|_{b}$ and $|v|_{c}=1$, or $\left|u_{1} \cdots u_{n-1}\right|_{a}+1=|v|_{a},\left|u_{1} \cdots u_{n-1}\right|_{b}-$ $2=|v|_{b}$ and $|v|_{d}=1$, i.e.,

$\Psi(v)=\Psi\left(u_{1} \cdots u_{n-1}\right)+(0,-1,1,0)$ or $\Psi(v)=\Psi\left(u_{1} \cdots u_{n-1}\right)+(1,-2,0,1)$,

then we say that $u$ satisfies property (SD.2).

A factor $u=u_{1} \cdots u_{n} \in\{a, b\}^{*}$ of $\mathbf{w}_{-}$is suffix-disqualifying, if either,

- for all $j \in\{1, \ldots, n\}$, the suffixes $u_{j} \cdots u_{n}$ all satisfy property (SD.1), or

- for all $j \in\{1, \ldots, n-1\}$, the suffixes $u_{j} \cdots u_{n}$ all satisfy property (SD.2).

Example 43. Assume that $\mathbf{w}_{-}=a a b c d a a a b a c \cdots$. The prefix $a a b$ is suffixdisqualifying. Indeed, the factors $a, a a$ and $a a a$ occurring in $\mathbf{w}_{-}$show that the suffixes $b, a b$ and $a a b$ satisfy (SD.1). Note that the factor $a b a$ satisfies (SD.2), because its prefix of length 2 contains exactly one $a$ and one $b$, but the factor $a c$ contains the same number of $a$ 's and one $b$ has been replaced with $c$.

Let $u \in \operatorname{Fac}\left(\mathbf{w}_{-}\right) \cap\{a, b\}^{*}$. Note that, if all suffixes of $u$ satisfy (SD.1), then $u$ ends with $b$.

Definition 44. A 4-tuple $(\alpha, \beta, \gamma, \delta)$ satisfies the combinatorial admissibility test (or, is CAT) if

- $\gamma>0$ and for all prefixes $p=w_{0} w_{1} \cdots w_{n}$ of $\mathbf{w}_{+}$such that $2 \leq|p|<B_{1}$, i.e., $1 \leq n \leq B_{1}-2$, with $w_{i} \in\{a, b\}$ for $i=1, \ldots, n, p$ is not disqualifying ${ }^{+}$.

- $\gamma<0$ and, for any prefix $p \in\{a, b\}^{*}$ of $\mathbf{w}_{-}$of length less than $B_{1}, p$ is not suffix-disqualifying.

Hence the word given in Example 43 corresponds to a 4-tuple which is not CAT (if we suppose $B_{1}$ is large enough). It will be shown in the next Section that such tuples correspond to sets that are not admissible. Indeed, since $a a b$ is suffix-disqualifying, it suffices to consider the position derived from the factor aaa, i.e., $\left(\pi_{1}(a)+\pi_{1}(a)+\pi_{1}(a), \pi_{2}(a)+\pi_{2}(a)+\pi_{2}(a)\right)$. From this position (which is not in $\mathcal{P})$, the only available moves to a position in $\mathcal{P}$ would be to go either to $\left(\pi_{1}(a)+\pi_{1}(a), \pi_{2}(a)+\pi_{2}(a)\right)$, to $\left(\pi_{1}(a), \pi_{2}(a)\right)$ or to $(0,0)$. But such moves are forbidden since the factors $a, a a$ and $a a a$ appear in $\mathbf{w}_{-}$. 


\section{Equivalence Between CAT And admissible sets}

With Definitions 44 and 2, we state the first part of the result.

Theorem 45. If the 4-tuple $(\alpha, \beta, \gamma, \delta)$ is not $C A T$, then $\mathcal{P}$ is not admissible.

Proof. Assume that $\gamma<0$ and there exists a prefix $p=w_{0} \cdots w_{n-1} \in\{a, b\}^{*}$ of $\mathbf{w}_{-}$of length $n$ less than $B_{1}$ such that $p$ is suffix-disqualifying. Therefore, since $\pi_{1}(a)=\pi_{1}(b)=1$, the first $n+1$ elements in $\mathcal{P}$ are $\left(i, B_{i}\right)$ for $i=0, \ldots, n$.

Assume first that all the suffixes of $p$ satisfy (SD.1). Consider the position $\left(n, B_{n}-1\right)$ which is not in $\mathcal{P}$. We will show that from $\left(n, B_{n}-1\right)$, there is no allowed move leading to a position in $\mathcal{P}$. Proceed by contradiction. Assume that there exists $i<n$ such that we can play the move $\left(n, B_{n}-1\right) \rightarrow\left(A_{i}, B_{i}\right)$. It implies that $\left(n-A_{i}, B_{n}-B_{i}-1\right)$ is not in $\mathcal{P}-\mathcal{P}$. By assumption, the suffix $w_{i} \cdots w_{n-1}$ satisfies (SD.1). Hence there exists a factor $f=w_{j+i} \cdots w_{j+n-1} \in\{a, b\}^{*}$ in $w$ of length $n-i=n-A_{i}$ such that $|f|_{b}<\left|w_{i} \cdots w_{n-1}\right|_{b}$. Since $\pi_{1}(a)=\pi_{1}(b)=1$, we get $A_{j+n}-A_{j+i}=\sum_{k=i}^{n-1} \pi_{1}\left(w_{j+k}\right)=n-i$. Note that $\pi_{2}(f)$ and $\pi_{2}\left(w_{i} \cdots w_{n-1}\right)$ are factors of the Sturmian word $\mathbf{s}_{\beta, \delta}$. Hence, the balance property implies that $|f|_{b}-1=\left|w_{i} \cdots w_{n-1}\right|_{b}$. From this, it follows that

$$
B_{j+n}-B_{j+i}=\sum_{k=i}^{n-1} \pi_{2}\left(w_{j+k}\right)=\sum_{k=i}^{n-1} \pi_{2}\left(w_{k}\right)-1=B_{n}-B_{i}-1
$$

and $\left(A_{j+n}-A_{j+i}, B_{j+n}-B_{j+i}\right)=\left(n-A_{i}, B_{n}-B_{i}-1\right)$ is in $\mathcal{P}-\mathcal{P}$.

Assume now that all the suffixes of $p$ satisfy (SD.2). One can proceed in a similar way to prove that from the position $\left(n, B_{n-1}-1\right)$ which is not in $\mathcal{P}$, there is no allowed move leading to a position in $\mathcal{P}$.

Assume that $\gamma>0$ and there exists a prefix $p=w_{0} w_{1} \cdots w_{n-1}$ of $\mathbf{w}_{+}$such that $2 \leq|p|<B_{1}, w_{1} \cdots w_{n-1} \in\{a, b\}^{*}$ and $p$ is disqualifying ${ }^{+}$. Therefore, the first $n+1$ elements in $\mathcal{P}$ are $\left(i, B_{i}\right)$ for $i=0, \ldots, n$ (see above). Since $p$ is disqualifying ${ }^{+}$, from Definition 41, $B_{n}-B_{n-1}=\pi_{2}\left(w_{n-1}\right) \in 2 B_{1}+\{-1,0,1\}$ and $B_{1} \geq 3$. Hence $B_{n-1}+B_{1}<B_{n}$ and $\left(n, B_{n-1}+B_{1}\right)$ is not in $\mathcal{P}$. We will show that from this position $\left(n, B_{n-1}+B_{1}\right)$, there is no allowed move leading to a position in $\mathcal{P}$. We proceed by contradiction. Assume first that there is a move $\left(n, B_{n-1}+B_{1}\right) \rightarrow(0,0)$. Since $p$ is disqualifying ${ }^{+}$, there exists a factor $f=f_{1} \cdots f_{n-1}$ occurring in $\mathbf{w}_{+}$ such that $\sum_{k=1}^{n-1} f_{k}=\left(n, B_{n-1}+B_{1}\right)$. In other words, $\left(n, B_{n-1}+B_{1}\right)$ belongs to $\mathcal{P}-\mathcal{P}$. Now assume that there is a move $\left(n, B_{n-1}+B_{1}\right) \rightarrow\left(A_{j}, B_{j}\right)$, with $j>0$. Since $p$ is disqualifying ${ }^{+}, w_{j} \cdots w_{n-2}$ is the reversal of $w_{1} \cdots w_{n-j-1}$. Hence we get $\sum_{k=j}^{n-2} \pi_{2}\left(w_{k}\right)+B_{1}=\sum_{k=1}^{n-j-1} \pi_{2}\left(w_{k}\right)+\pi_{2}\left(w_{0}\right)=B_{n-1}+B_{1}-B_{j}$ and $\left(n-j, B_{n-1}+B_{1}-B_{j}\right)$ belongs to $\mathcal{P}-\mathcal{P}$.

We now turn to the converse. We show that, if the 4 -tuple $(\alpha, \beta, \gamma, \delta)$ is CAT, then an invariant game having $\mathcal{P}$ as set of $P$-positions exists, i.e., $\mathcal{P}$ is admissible. As mentioned in Remark 3, it suffices to test the maximal subtraction game having

$$
\mathcal{M}:=\mathbb{N}^{2} \backslash(\mathcal{P}-\mathcal{P})
$$

as set of moves. The next result is the analogue of Lemma 9 for homogeneous Beatty sequences.

Lemma 46. If the 4-tuple $(\alpha, \beta, \gamma, \delta)$ is $C A T$, then we have

$$
\{(k, 0),(0, k) \mid k \geq 1\} \cup\left\{(1, k) \mid 1 \leq k<B_{1}\right\} \subseteq \mathcal{M} .
$$


Proof. Since $\left\{A_{n} \mid n>0\right\}$ and $\left\{B_{n} \mid n>0\right\}$ make a partition of $\mathbb{N}$, we clearly have that $(k, 0)$ and $(0, k)$ belong to $\mathcal{M}$.

Let $k$ be such that $1 \leq k<B_{1}$. By way of contradiction, assume that $(1, k) \in$ $\mathcal{P}-\mathcal{P}$. Note that $\delta<1$ (see (11)), implies $B_{1}=\lfloor\beta+\delta\rfloor \leq\lfloor\beta\rfloor+1$. We consider two cases:

- $(1, k)=\left(A_{n}-A_{m}, B_{n}-B_{m}\right)$ for some $n>m \geq 0$. According to Lemma 35, $A_{n}-A_{m}=1$ implies $n=m+1$ and $\left(A_{n}-A_{m}, B_{n}-B_{m}\right)$ can possibly take the values $\left(1, B_{1}\right),(1,\lfloor\beta\rfloor)$ and $(1,\lfloor\beta\rfloor+1)$. Since $k<B_{1}$, if $k<\lfloor\beta\rfloor$, then $(1, k)$ cannot be of this form. If $k=\lfloor\beta\rfloor$, then $k<B_{1}$ implies that $B_{1}=\lfloor\beta\rfloor+1$ and thus $\gamma<0$. In other words, $b$ is a prefix of $\mathbf{w}_{-}$. By assumption, $(\alpha, \beta, \gamma, \delta)$ is CAT (and we are in the situation where $\gamma<0)$, this means that $\mathbf{w}_{-}$contains no occurrence of $a$ (coding the difference $(1,\lfloor\beta\rfloor)$ ) because otherwise, the prefix $b$ of $\mathbf{w}_{-}$would satisfy (SD.1).

- $(1, k)=\left(A_{n}-B_{m}, B_{n}-A_{m}\right)$ for some $n>m \geq 0$. Hence we have $B_{n}-A_{m}=$ $\left(B_{n}-B_{m}\right)+\left(B_{m}-A_{m}\right) \geq B_{1}$, contradicting the hypothesis.

Lemma 47. Let $n, m, k, l, \Delta$ be five integers such that $n>m \geq 0, k>l \geq 0$, and $\Delta \geq 2$. If $A_{n}-A_{m}=A_{k}-A_{l}+\Delta$, then we have $B_{n}-B_{m}>B_{k}-B_{l}+\Delta-4$.

Proof. Consider the relative position of the straight line of equation $y=\frac{\beta}{\alpha} x+2 \delta$ and the point of coordinates $\left(A_{n}, B_{n}\right)$. More precisely, consider the difference of $y$-coordinates between the point of the line having a $x$-coordinate equal to $A_{n}$ and the point $\left(A_{n}, B_{n}\right)$ :

$$
\frac{\beta}{\alpha} A_{n}+2 \delta-B_{n}=\frac{\beta}{\alpha}\lfloor n \alpha+\gamma\rfloor+2 \delta-\lfloor n \beta+\delta\rfloor .
$$

This quantity is equal to

$\frac{\beta}{\alpha}(n \alpha+\gamma)+2 \delta-(n \beta+\delta)-\frac{\beta}{\alpha}\{n \alpha+\gamma\}+\{n \beta+\delta\}=\beta-\frac{\beta}{\alpha}\{n \alpha+\gamma\}+\{n \beta+\delta\}$

where, for the last equality, we have used (4). Hence, this difference satisfies

$$
\frac{\beta}{\alpha} A_{n}+2 \delta-B_{n} \in\left(\beta-\frac{\beta}{\alpha}, \beta+1\right) \text {, i.e., } \frac{\beta}{\alpha} A_{n}+2 \delta-\beta-1<B_{n}<\frac{\beta}{\alpha} A_{n}+2 \delta-\beta+\frac{\beta}{\alpha} \text {. }
$$

Assume that $A_{k}-A_{l}=i$. From the above computation, we get

$$
B_{k}-B_{l}<\frac{\beta}{\alpha} A_{k}+2 \delta+\frac{\beta}{\alpha}-\left(\frac{\beta}{\alpha} A_{l}+2 \delta-1\right)=(i+1) \frac{\beta}{\alpha}+1 .
$$

Similarly, by assumption $A_{n}-A_{m}=i+\Delta$ and we get

$$
B_{n}-B_{m}>\frac{\beta}{\alpha} A_{n}+2 \delta-1-\left(\frac{\beta}{\alpha} A_{m}+2 \delta+\frac{\beta}{\alpha}\right)=(i+\Delta-1) \frac{\beta}{\alpha}-1 .
$$

Hence we get

$$
\begin{aligned}
B_{n}-B_{m}-\left(B_{k}-B_{l}\right)-\Delta+4 & >(i+\Delta-1) \frac{\beta}{\alpha}-1-(i+1) \frac{\beta}{\alpha}-1-\Delta+4 \\
& =(\Delta-2)\left(\frac{\beta}{\alpha}-1\right) \\
& \geq 0
\end{aligned}
$$

Observe that this last inequality holds since $\Delta \geq 2$ and $\alpha<\beta$. 
We will make use of Lemma 47 in the proof of Theorem 50 (and in particular, with the case (3.1.a)). Since our assumption is that $B_{1} \geq 3$, the condition $\Delta \geq 2$ will be fulfilled. Therefore, in order to hypothetically prove our main theorem in the case $B_{1}=2$, another argument should be developed. See the concluding Section 8 .

Lemma 48. The map $n \mapsto B_{n}-A_{n}$ is non-decreasing.

Proof. Since $A_{n+1}-A_{n} \in\{1,2\}, B_{n+1}-B_{n} \in\{\lfloor\beta\rfloor,\lfloor\beta\rfloor+1\}$, then $B_{n+1}-A_{n+1} \geq$ $B_{n}-A_{n}+\lfloor\beta\rfloor-2$ and the conclusion follows from the fact that $\lfloor\beta\rfloor \geq 2$.

Lemma 49. If the 4-tuple $(\alpha, \beta, \gamma, \delta)$ is $C A T$ and $\lfloor\beta\rfloor=2$, then the following properties hold:

(a) $B_{1}=3$.

(b) $\gamma<0$.

(c) $\mathbf{w}_{-}$starts with $b b$. The factor a never appears in $\mathbf{w}_{-}$.

(d) The first three pairs of the sequence $\left(A_{n}, B_{n}\right)$ are $(0,0),(1,3),(2,6)$.

(e) The factor cc never occurs in $\mathbf{w}_{-}$.

Proof. $\quad$ (a) Obtained from $B_{1} \geq 3$ and $\delta<1$.

(b) Since $B_{1}>\lfloor\beta\rfloor$, we have $\delta>0$, and thus $\gamma<0$.

(c) Since $\left(A_{1}, B_{1}\right)=(1,3)$, it means that $w_{0}=b$ and we necessarily have $A_{2}=2$. Since $(\alpha, \beta, \gamma, \delta)$ is CAT, the prefix $b$ is not suffix-disqualifying, meaning that $a$ never appears in $\mathbf{w}_{-}$. In other words, $w_{1}=b$.

(d) Directly deduced from the previous item.

(e) Since $b b$ occurs in $\mathbf{w}_{-}$and $\pi_{2}\left(\mathbf{w}_{-}\right)$is Sturmian, it means that $c c$ never appears.

Theorem 50. If the 4-tuple $(\alpha, \beta, \gamma, \delta)$ is $C A T$, then the invariant game having $\mathcal{M}$ as set of moves admits $\mathcal{P}$ as set of $P$-positions, i.e., $\mathcal{P}$ is admissible.

Proof. By construction of $\mathcal{M}$, it is clear that from a position in $\mathcal{P}$ any move leads to a position in $\mathcal{N}=\mathbb{N}^{2} \backslash \mathcal{P}$. Now we show that if $(x, y)$ belongs to $\mathcal{N}$, there exists a move $m \in \mathcal{M}$ such that $(x, y)-m$ belongs to $\mathcal{P}$. If $x=0$ or $y=0$, we conclude directly using Lemma 46. Without loss of generality, we now may assume that $0<x \leq y$.

Since $\left\{A_{n} \mid n>0\right\}$ and $\left\{B_{n} \mid n>0\right\}$ make a partition of $\mathbb{N}$, we consider three cases.

Case 1) If $x=B_{i}$ for some $i>0$, then consider the move $\left(0, y-A_{i}\right)$. From Lemma 46, this move belongs to $\mathcal{M}$ since $y \geq x=B_{i}>A_{i}$. Hence the resulting position $(x, y)-\left(0, y-A_{i}\right)=\left(B_{i}, A_{i}\right)$ belongs to $\mathcal{P}$.

Case 2) If $x=A_{i}$ and $y>B_{i}$, then consider the move $\left(0, y-B_{i}\right) \in \mathcal{M}$ leading to the position $\left(A_{i}, B_{i}\right)$.

Case 3) Consider the case where $x=A_{i} \leq y<B_{i}$. Note that we do not take into account the case $y=B_{i}$, since we would have $(x, y)=\left(A_{i}, B_{i}\right)$ which does not belong to $\mathcal{N}$. We consider two sub-cases:

Case 3.1) $B_{1}<x=A_{i} \leq y<B_{i}$.

3.1.a) If $y-x \geq 2$, we aim to show that it is always possible to move either to $\left(1, B_{1}\right)$ or to $\left(B_{1}, 1\right)$. Since the move should not belong to $\mathcal{P}-\mathcal{P}$, we have three situations that may occur. 
Situation 1. We start by proving that these two moves are not of the form $\left(A_{n}-B_{m}, B_{n}-A_{m}\right)$ with $n>m>0$. First assume that $\left(A_{i}-1, y-B_{1}\right)=$ $\left(A_{n}-B_{m}, B_{n}-A_{m}\right)$ for some $n>m>0$. Hence we have

$$
A_{i}-1=A_{n}-B_{m} \quad \text { and } \quad y-B_{1}=B_{n}-A_{m} .
$$

In that case, since $A_{n}-A_{i}=B_{m}-1 \geq 0$, note that we necessarily have $i \leq n$. We now subtract the previous two equalities to obtain

$$
y=B_{n}-A_{n}+B_{m}-A_{m}+A_{i}-1+B_{1}
$$

And since $y<B_{i}$, the following inequality holds

$$
B_{n}-A_{n}+B_{m}-A_{m}<B_{i}-A_{i}+A_{1}-B_{1}
$$

Since $n \geq i$, the contradiction is guaranteed thanks to Lemma 49 and $A_{1}-B_{1}<0$.

Now suppose that $\left(A_{i}-B_{1}, y-1\right)=\left(A_{n}-B_{m}, B_{n}-A_{m}\right)$ for some $n>m>0$. With the same argument as above, we get

$$
B_{n}-A_{n}+B_{m}-A_{m}<B_{i}-A_{i}+B_{1}-A_{1}
$$

Since we still have $n \geq i$ in that case $\left(A_{n}-A_{i}\right.$ being positive), and $m \geq 1$, the contradiction is ensured thanks to Lemma 49

Situation 2. Now assume that $\left(A_{i}-1, y-B_{1}\right)=\left(B_{n}-A_{m}, A_{n}-B_{m}\right)$ for some $n>m>0$. Again, subtracting the two equalities, we get

$$
y-A_{i}=B_{1}-1-\left(B_{m}-A_{m}\right)-\left(B_{n}-A_{n}\right) .
$$

The left hand side is non-negative, but thanks to Lemma 49, the right hand side is negative. Similarly, if $\left(A_{i}-B_{1}, y-1\right)=\left(B_{n}-A_{m}, A_{n}-B_{m}\right)$ for some $n>m>0$, we get directly $y-A_{i}=1-B_{1}-\left(B_{m}-A_{m}\right)-\left(B_{n}-A_{n}\right)$. This is a contradiction because the l.h.s. is non-negative and the r.h.s. is negative.

Situation 3. It now remains to prove that at least one of the two moves is neither of the form $\left(A_{n}-A_{m}, B_{n}-B_{m}\right)$, nor $\left(B_{n}-B_{m}, A_{n}-A_{m}\right)$ with $n>m \geq 0$.

Case 1: $A_{i}-1 \leq y-B_{1}$. From Lemma 35. $B_{n}-B_{m} \geq(n-m)\lfloor\beta\rfloor$ and $A_{n}-A_{m} \leq$ $2(n-m)$. Hence $\left(A_{i}-1, y-B_{1}\right)$ cannot be of the form $\left(B_{n}-B_{m}, A_{n}-A_{m}\right)$, except in the particular case where $A_{i}-1=y-B_{1}$ and $B_{n}-B_{m}=A_{n}-A_{m}$. Again from Lemma 35, this may only happen if $\lfloor\beta\rfloor=2$, together with $A_{n}-A_{m}=2(n-m)$ and $B_{n}-B_{m}=(n-m)\lfloor\beta\rfloor$. But from Lemma 49, the factor $c c$ never occurs in $\mathbf{w}_{-}$ in that case, implying $n-m=1$, then $A_{i}=3$, contradicting $A_{i}>B_{1}$.

Hence $\left(A_{i}-1, y-B_{1}\right)$ can only be of the form $\left(A_{n}-A_{m}, B_{n}-B_{m}\right)$. If it is the case, we will show that the move $\left(A_{i}-B_{1}, y-1\right)$ is not forbidden. By way of contradiction, assume that both moves are forbidden, i.e., there exists $n>m \geq 0$ and $k>l \geq 0$ satisfying

$$
\begin{gathered}
x-1=A_{n}-A_{m} \quad \text { and } \quad y-B_{1}=B_{n}-B_{m}, \\
x-B_{1}=A_{k}-A_{l} \quad \text { and } \quad y-1=B_{k}-B_{l} .
\end{gathered}
$$

Indeed, since $A_{i}-B_{1}<y-1$, then $\left(A_{i}-B_{1}, y-1\right)$ cannot be of the form $\left(B_{k}-B_{l}, A_{k}-A_{l}\right)$ because $B_{k}-B_{l} \geq A_{k}-A_{l}$. Moreover, the other forms $\left(A_{k}-\right.$ $\left.B_{l}, B_{k}-A_{l}\right)$ or $\left(B_{k}-A_{l}, A_{k}-B_{l}\right)$ are also excluded thanks to the above discussion in situations 1 and 2 . Thus, if $\left(A_{i}-B_{1}, y-1\right)$ is forbidden, it can only be of the form $\left(A_{k}-A_{l}, B_{k}-B_{l}\right)$.

Now, by subtracting the last two equalities from the first two ones, we obtain

$$
A_{n}-A_{m}=A_{k}-A_{l}+B_{1}-1 \text { and } B_{n}-B_{m}=B_{k}-B_{l}-\left(B_{1}-1\right) \text {. }
$$


Since $B_{1}-1 \geq 2$, Lemma 47 with $\Delta=B_{1}-1$ yields a contradiction.

Case 2: $A_{i}-1>y-B_{1}$. Hence $\left(A_{i}-1, y-B_{1}\right)$ can only be of the form $\left(B_{n}-B_{m}, A_{n}-A_{m}\right)$. Similarly, $\left(A_{i}-B_{1}, y-1\right)$ satisfies $A_{i}-B_{1}<y-1$, and thus can only be of the form $\left(A_{n}-A_{m}, B_{n}-B_{m}\right)$. Now assume that both moves are forbidden, i.e., there exists $n>m \geq 0$ and $k>l \geq 0$ satisfying

$$
\begin{gathered}
x-1=B_{n}-B_{m} \quad \text { and } \quad y-B_{1}=A_{n}-A_{m}, \\
y-1=B_{k}-B_{l} \quad \text { and } \quad x-B_{1}=A_{k}-A_{l} .
\end{gathered}
$$

By subtracting the last two equalities from the first two ones, we obtain

$$
A_{n}-A_{m}=A_{k}-A_{l}+y-x \quad \text { and } \quad B_{n}-B_{m}=B_{k}-B_{l}-(y-x) .
$$

Since $y-x \geq 2$, Lemma 47 with $\Delta=y-x$ yields a contradiction.

3.1.b) If $y-x<2$, we will show that playing from $(x, y)=\left(A_{i}, y\right)$ to $(0,0)$ is "almost always" legal. Indeed, if this move was forbidden, there would exist a factor $f_{1} \cdots f_{l}$ in $\mathbf{w}$, of length $l>1$ (since $A_{i}>B_{1} \geq 3$ ), and satisfying $y-x=$ $\sum_{k=1}^{l} \pi_{2}\left(f_{k}\right)-\sum_{k=1}^{l} \pi_{1}\left(f_{k}\right)<2$. According to Lemma 35, the only factors that may satisfy these conditions need to have $\lfloor\beta\rfloor=2$ and are:

- factors of the kind $c c^{+}$: According to Lemma 49, $c c$ never occur in $\mathbf{w}_{-}$, which excludes such factors.

- factors of the kind $a c^{+}$and their permutations: According to Lemma 49 these factors never occur because they contain $a$.

- factors of the kind $d c^{+}$and their permutations: According to Lemma 49, since $c c$ never occur, the list reduces to the factors $\{d c, c d, c d c\}$. The case $c d c$ would lead to study $\left(A_{i}, A_{i}+1\right)=(6,7)$, which is impossible since $B_{2}=6$ from Lemma 35. The cases $c d$ and $d c$ lead to examine position $\left(A_{i}, A_{i}+1\right)=(4,5)$. For this particular case, play $(4,5) \rightarrow(3,1)$, which is legal since $(1,4)=(1,\lfloor\beta\rfloor+2)$ is clearly not in $\mathcal{P}-\mathcal{P}$ from Lemma 35.

Case 3.2) $x=A_{i} \leq y<B_{i}$ and $A_{i}<B_{1}$. If $i=1$, then $(x, y)=(1, y)$ and thanks to Lemma 46, playing to $(0,0)$ is allowed. Hence in the following discussion, we may assume that $i>1$. Since $A_{i}<B_{1}$ and the increasing sequences $\left(A_{n}\right)_{n>0}$ and $\left(B_{n}\right)_{n>0}$ make a partition of $\mathbb{N}_{>0}$, we have $A_{i^{\prime}}=i^{\prime}$ for $0<i^{\prime} \leq i$.

If $y=B_{j}$ for some $j<i$, playing to $\left(A_{j}, B_{j}\right)$ is allowed from Lemma 46 .

3.2.a) If $y<B_{i-2}$, there exists $t \geq 3$ such that $B_{i-t}<y<B_{i-t+1}$ and $i \geq 3$. Playing to $\left(A_{i-t}, B_{i-t}\right)$ is allowed. Indeed, we have

$$
(x, y)-\left(A_{i-t}, B_{i-t}\right)=(t, k) \text { where } t \geq 3, k \leq\lfloor\beta\rfloor
$$

and the conclusion follows from Lemma 35. $(t, k)$ with $t \geq 3, k \leq\lfloor\beta\rfloor$ is not of the form $\left(A_{n}-A_{m}, B_{n}-B_{m}\right)$. In addition, it is easy to see that $(t, k)$ can neither be of the form $\left(A_{n}-B_{m}, B_{n}-A_{m}\right)$ (otherwise we would have $k=B_{n}-A_{m} \geq\lfloor\beta\rfloor+1$ ), nor of the form $\left(B_{n}-A_{m}, A_{n}-B_{m}\right)$ (otherwise we would have $t=B_{n}-A_{m} \geq B_{1}$, contradicting the property $\left.t=A_{i}-A_{i-t}<B_{1}\right)$, nor $\left(B_{n}-B_{m}, A_{n}-A_{m}\right)$ (otherwise we would have $B_{n}-B_{m}=t<i=A_{i}<B_{1}$, implying $B_{n}-B_{m}<\lfloor\beta\rfloor$ !)

3.2.b) If $B_{i-2}<y<B_{i-1}-1$ or if $y=B_{i-1}-1$ and $B_{i-1}-B_{i-2}=\lfloor\beta\rfloor$, then playing to $\left(A_{i-2}, B_{i-2}\right)$ is allowed. Indeed, we have

$$
(x, y)-\left(A_{i-2}, B_{i-2}\right)=(2, k) \text { where } k<\lfloor\beta\rfloor
$$

and, as in the previous case, the conclusion follows again from Lemma 35 . 
3.2.c) If $y=B_{i-1}-1$ and $B_{i-1}-B_{i-2}=\lfloor\beta\rfloor+1$, we consider two cases according to the sign of $\gamma$.

$\gamma>0$ : We will show that moving to $(0,0)$ is always possible. For this purpose, it suffices to show that there exists no factor $f=f_{1} \cdots f_{l}$ of $\mathbf{w}_{+}$such that $\sum_{k=1}^{l} f_{k}=\left(i, B_{i-1}-1\right)$. Assume that such a factor $f$ exists. In other words, $\left(i, B_{i-1}-1\right)$ is of the kind $\left(A_{n}-A_{n-l}, B_{n}-B_{n-l}\right)$ and belongs to $\mathcal{P}-\mathcal{P}$. Necessarily the length of $f$ satisfies $l \leq i$ (because $\left.\pi_{1}(f) \in\{1,2\}^{*}\right)$.

If $l=i$, since $s_{\beta, \delta}(0) w_{1} w_{2} \cdots$ is a Sturmian word, one can use Remark 29 and the fact that $s_{\beta, \delta}(0)>\pi_{2}\left(w_{0}\right)$, to get

$$
B_{i-1}-1=\sum_{k=1}^{l} \pi_{2}\left(f_{k}\right) \geq \sum_{k=0}^{i-1} \pi_{2}\left(w_{k}\right)
$$

As (12) gives $\sum_{k=0}^{i-1} \pi_{2}\left(w_{k}\right)=B_{i-1}+\pi_{2}\left(w_{i-1}\right)$, we get a contradiction.

If $l=i-1$, we conclude in the same way that $\sum_{k=1}^{l} \pi_{2}\left(f_{k}\right) \geq \sum_{k=0}^{i-2} \pi_{2}\left(w_{k}\right)=$ $B_{i-1}$, a contradiction.

If $l<i-1$, then using Remark 29, we get

$\sum_{k=1}^{l} \pi_{2}\left(f_{k}\right) \leq \sum_{k=0}^{i-3} \pi_{2}\left(s_{\beta, \delta}(k)\right)+1=\pi_{2}\left(w_{0}\right)-\lfloor\delta\rfloor+\sum_{k=1}^{i-3} \pi_{2}\left(s_{\beta, \delta}(k)\right)+1 \leq B_{i-2}+2$

where for the last inequality, we have used the fact that $\lfloor\delta\rfloor \leq-1$. Recall that we are assuming here that $B_{i-1}-B_{i-2}=\lfloor\beta\rfloor+1$ (but we also have $\lfloor\beta\rfloor \geq 3$ since $\delta<0$ and $\left.B_{1} \geq 3\right)$. Hence, $\sum_{k=1}^{l} \pi_{2}\left(f_{k}\right) \leq B_{i-2}+2<B_{i-1}-1$ which is again a contradiction.

$\gamma<0$ : Consider the prefix $p$ of $\mathbf{w}_{-}$of length $i$. Since $A_{j}-A_{j-1}=1$ for all $0<j \leq i$, we know that $p \in\{a, b\}^{*}$ and $|p|<B_{1}$. Since $(\alpha, \beta, \gamma, \delta)$ is CAT, the prefix $p$ is not suffix-disqualifying. In particular, it means that there exists some $j \in\{0, \ldots, i-2\}$ such that $w_{j} \cdots w_{i-1}$ does not satisfy property (SD.2). Playing from $(x, y)$ to $\left(A_{j}, B_{j}\right)$ is thus allowed. Indeed, assume on the contrary that $\left(x-A_{j}, y-B_{j}\right)$ belongs to $\mathcal{P}-\mathcal{P}$ and is of the form $\left(A_{n}-A_{m}, B_{n}-B_{m}\right)$ (the other case $\left(A_{m}-B_{n}, B_{m}-A_{n}\right)$ cannot occur). It would mean that there exists a factor $f=f_{1} \cdots f_{l}$ of $\mathbf{w}_{-}$such that $\sum_{k=1}^{l} f_{k}=\left(i-j, B_{i-1}-1-B_{j}\right)$. Necessarily we have $l \leq i-j$. If $l=i-j$, then $\sum_{k=1}^{l} \pi_{2}\left(f_{k}\right) \geq \sum_{k=j}^{i-1} \pi_{2}\left(w_{k}\right)-1$, since $\pi_{2}\left(\mathbf{w}_{-}\right)$is Sturmian. But we also have $\sum_{k=1}^{l} \pi_{2}\left(f_{k}\right)=B_{i-1}-1-B_{j}=\sum_{k=j}^{i-1} \pi_{2}\left(w_{k}\right)-1$, leading to a contradiction. If $l<i-j-1$, then $\sum_{k=1}^{l} \pi_{2}\left(f_{k}\right) \leq \sum_{k=j}^{i-3} \pi_{2}\left(w_{k}\right)+1$. But we also have $\sum_{k=1}^{l} \pi_{2}\left(f_{k}\right)=B_{i-1}-1-B_{j}=\sum_{k=j}^{i-1} \pi_{2}\left(w_{k}\right)-1$, leading to a contradiction. Hence $l=i-j-1$, meaning that $f$ is a factor of $\mathbf{w}_{-}$over $\{a, b, c, d\}^{*}$ of length $(i-j-1)$ with exactly $|f|_{c}+|f|_{d}=1$. In particular, $\sum_{k=1}^{l} \pi_{1}\left(f_{k}\right)=(i-j)$. Assume first that $|f|_{c}=1$. Since $w_{j} \cdots w_{i-1}$ does not satisfy property (SD.2), we get $|f|_{a}=\left|w_{j} \cdots w_{i-2}\right|_{a}-1,|f|_{b}=\left|w_{j} \cdots w_{i-2}\right|_{b}$,

\footnotetext{
${ }^{1}$ In all what follows, the case $\left(A_{n}-B_{m}, B_{n}-A_{m}\right)$ is easy to consider. Indeed, here we get from $\left(A_{i}, B_{i-1}-1\right)=\left(A_{n}-B_{m}, B_{n}-A_{m}\right)$ that $A_{n}-A_{i}=B_{m}$ and $B_{n}-B_{i}-1=A_{m}-1$, thus $B_{n}-B_{i} \leq A_{m}-4$ and $A_{n}-A_{i}>B_{n}-B_{i}$ which is not possible. Note that we do not have to consider moves $\left(B_{n}-B_{m}, A_{n}-A_{m}\right)$ nor $\left(B_{n}-A_{m}, A_{n}-B_{m}\right)$ because $B_{n}-B_{m} \geq A_{n}-A_{m}$ and $B_{n}-A_{m} \geq A_{n}-B_{m}$ but we are in a position $(i, y)$ with $y<B_{i}$. To avoid lengthy discussions, we have only considered the more intricate situation.
} 
and $\sum_{k=1}^{l} \pi_{2}\left(f_{k}\right)=\sum_{k=j}^{i-2} \pi_{2}\left(w_{k}\right)$, which is a contradiction. The proof is similar in the case $|f|_{d}=1$.

3.2.d) If $B_{i-1}<y<B_{i}-1$ or if $y=B_{i}-1$ and $B_{i}-B_{i-1}=\lfloor\beta\rfloor$, then consider the move

$$
(x, y)-\left(A_{i-1}, B_{i-1}\right)=(1, k) \text { where } k<\lfloor\beta\rfloor .
$$

$\gamma<0$ : This move is allowed since $(1, k)$ never occurs in $\mathbf{w}_{-}$.

$\gamma>0$ : This move is allowed whenever $k \neq B_{1}$ (recall that $\left(1, B_{1}\right)$ occurs as $w_{0}$ in $\left.\mathbf{w}_{+}\right)$. Now assume that $y=B_{i-1}+B_{1}$. In particular, we get $B_{1} \leq\lfloor\beta\rfloor-1$.

In the case where $w_{1} \cdots w_{i-2}$ is a palindrome, we show that we can play to $(0,0)$. Assume to the contrary that there exists a factor $f=f_{1} \cdots f_{l}$ occurring in $\mathbf{w}_{+}$such that $\sum_{k=1}^{l} f_{i}=(x, y)$. Necessarily, $l \leq i$. If $l=i$, then $\sum_{k=1}^{l} \pi_{2}\left(f_{k}\right) \geq \sum_{k=1}^{i-2} \pi_{2}\left(w_{k}\right)+2\lfloor\beta\rfloor$ since $\mathbf{s}_{\beta, \delta}$ is Sturmian. But we also have $\sum_{k=1}^{l} \pi_{2}\left(f_{k}\right)=y=\sum_{k=1}^{i-2} \pi_{2}\left(w_{k}\right)+2 B_{1}$ contradicting the fact that $B_{1}<\lfloor\beta\rfloor$. If $l<i-1$, then $\sum_{k=1}^{l} \pi_{2}\left(f_{k}\right) \leq \sum_{k=1}^{i-2} \pi_{2}\left(w_{k}\right)+1$. But we also have $\sum_{k=1}^{l} \pi_{2}\left(f_{k}\right)=y=\sum_{k=1}^{i-2} \pi_{2}\left(w_{k}\right)+2 B_{1}$ leading to the contradiction $2 B_{1} \leq 1$.

Hence $l=i-1$ and using again the Sturmian property, we get $\sum_{k=1}^{i-1} \pi_{2}\left(w_{k}\right)-$ $1 \leq \sum_{k=1}^{l} \pi_{2}\left(f_{k}\right) \leq \sum_{k=1}^{i-1} \pi_{2}\left(w_{k}\right)+1$. But $\sum_{k=1}^{l} \pi_{2}\left(f_{k}\right)=y=\sum_{k=1}^{i-1} \pi_{2}\left(w_{k}\right)+$ $2 B_{1}-B_{i}+B_{i-1}$ leading to

$$
B_{i}-B_{i-1}-1 \leq 2 B_{1} \leq B_{i}-B_{i-1}+1 \text {. }
$$

The prefix $w_{0} \cdots w_{i-1}$ is not disqualifying ${ }^{+}$. According to Definition 41, the situation can be split into three cases. Assume first that $2 B_{1}=\pi_{2}\left(w_{i-1}\right)-1$. The factor $f$ of length $i-1$ is such that $\sum_{k=1}^{l} \pi_{1}\left(f_{k}\right)=i$, this implies that $|f|_{c}+|f|_{d}=1$. Moreover, $\sum_{k=1}^{l} \pi_{2}\left(f_{k}\right)=y=\sum_{k=1}^{i-1} \pi_{2}\left(w_{k}\right)+2 B_{1}-$ $\pi_{2}\left(w_{i-1}\right)$. Hence $\sum_{k=1}^{l} \pi_{2}\left(f_{k}\right)=\sum_{k=1}^{i-1} \pi_{2}\left(w_{k}\right)-1$ and the factor $f$ must satisfy $\left|w_{1} \cdots w_{i-2}\right|_{a}=|f|_{a},\left|w_{1} \cdots w_{i-2}\right|_{b}-1=|f|_{b},|f|_{c}=1$. This contradicts the fact that $w_{0} \cdots w_{i-1}$ is not disqualifying ${ }^{+}$. The last two cases where $2 B_{1}=\pi_{2}\left(w_{i-1}\right)$ and $2 B_{1}=\pi_{2}\left(w_{i-1}\right)+1$ are treated similarly.

If $w_{1} \cdots w_{i-2}$ is not a palindrome, consider the smallest $j$ such that $w_{1+j} \neq w_{i-2-j}$. We can play to $\left(A_{i-j-2}, B_{i-j-2}\right)$ except if there exists some factor $f=w_{t} \cdots w_{t+l-1}$ occurring in $\mathbf{w}_{+}$and satisfying

$$
\sum_{k=t}^{t+l-1} w_{k}=\sum_{k=i-j-2}^{i-2} w_{k}+\left(1, B_{1}\right) .
$$

Assume to conclude this part of the proof that we are in this latter situation.

Clearly we have that $l \leq j+2$. If $t=0$, since $\pi_{1}\left(w_{0}\right)=\cdots=\pi_{1}\left(w_{l-1}\right)=$ 1 , then $l=j+2$, and $\sum_{k=t}^{t+\bar{l}-1} \pi_{2}\left(w_{k}\right) \neq \sum_{k=i-j-2}^{i-2} \pi_{2}\left(w_{k}\right)+B_{1}$. If $t \neq 0$ and $l<j+2$, from Remark 29, we have $\sum_{k=t}^{t+l-1} \pi_{2}\left(w_{k}\right)-1 \leq \sum_{k=i-j-2}^{i-2} \pi_{2}\left(w_{k}\right)$. Hence, $\sum_{k=t}^{t+l-1} \pi_{2}\left(w_{k}\right)<\sum_{k=i-j-2}^{i-2} \pi_{2}\left(w_{k}\right)+B_{1}$. If $t \neq 0$ and $l=j+2$, from Remark 29], we have $\sum_{k=t}^{t+l-1} \pi_{2}\left(w_{k}\right) \geq \sum_{k=i-j-2}^{i-1} \pi_{2}\left(w_{k}\right)-1$ and as $\sum_{k=t}^{t+l-1} \pi_{2}\left(w_{k}\right)=\sum_{k=i-j-2}^{i-1} \pi_{2}\left(w_{k}\right)+B_{1}-\pi_{2}\left(w_{i-1}\right)$. Hence we obtain $B_{1} \geq$ $\pi_{2}\left(w_{i-1}\right)-1$, which is a contradiction if $B_{1} \neq\lfloor\beta\rfloor-1$ or if $\pi_{2}\left(w_{i-1}\right)=\lfloor\beta\rfloor+1$. In the case where $B_{1}=\lfloor\beta\rfloor-1$ and $\pi_{2}\left(w_{i-1}\right)=\lfloor\beta\rfloor$, since $B_{1} \geq 3$, we get $\lfloor\beta\rfloor \geq 4$. In this particular case, none of the three situations described in 
Definition 411 can occur. Indeed, we have $2 B_{1}=2\lfloor\beta\rfloor-2>\lfloor\beta\rfloor+1=$ $\pi_{2}\left(w_{i-1}\right)+1$. Hence one can use the strategy described above (i.e., playing to $(0,0))$, by acting as if $w_{1} \cdots w_{i-2}$ was a palindrome.

3.2.e) If $y=B_{i}-1$ and $\pi_{2}\left(w_{i-1}\right)=B_{i}-B_{i-1}=\lfloor\beta\rfloor+1$, we consider two cases.

$\gamma>0$ : We will show that moving to $(0,0)$ is always possible. For this purpose, it suffices to show that there exists no factor $f=f_{1} \cdots f_{l}$ of $\mathbf{w}_{+}$such that $\sum_{k=1}^{l} f_{k}=\left(i, B_{i}-1\right)$. Assume that such a factor $f$ exists. Necessarily $f$ satisfies $l \leq i$. If $l<i$, then $\sum_{k=1}^{l} \pi_{2}\left(f_{k}\right) \leq \sum_{k=0}^{i-2} \pi_{2}\left(w_{k}\right)+2=B_{i-1}+2$, yielding a contradiction. If $l=i$, we have $\sum_{k=1}^{l} \pi_{2}\left(f_{k}\right) \geq \sum_{k=1}^{i-1} \pi_{2}\left(w_{k}\right)-$ $1+\lfloor\beta\rfloor$ because $w_{1} w_{2} \cdots$ is Sturmian. Since we must have $y=B_{1}+$ $\sum_{k=1}^{i-1} \pi_{2}\left(w_{k}\right)-1=\sum_{k=1}^{l} \pi_{2}\left(f_{k}\right)$, it implies $B_{1} \geq\lfloor\beta\rfloor$. As $B_{1}<\lfloor\beta\rfloor+1$ when $\gamma>0$, we have $B_{1}=\lfloor\beta\rfloor$. We deduce that $s_{\beta, \delta}(0)=\lfloor\beta\rfloor+1$, and $\sum_{k=0}^{i-1} s_{\beta, \delta}(k)=\sum_{k=0}^{i-1} \pi_{2}\left(w_{k}\right)+1=B_{i}+1$. Since a Sturmian word is balanced, no factor of length $i$ gives a sum equal to $B_{i}-1$.

$\gamma<0$ : We consider the prefix $p$ of $\mathbf{w}$ of length $i$. Since $A_{j}-A_{j-1}=1$ for all $0<j \leq i$, we know that $p \in\{a, b\}^{*}$ and $|p|<B_{1}$. Since $(\alpha, \beta, \gamma, \delta)$ is CAT, the prefix $p$ is not suffix-disqualifying. In particular, it means that there exists some $j \in\{0, \ldots, i-1\}$ such that $w_{j} \cdots w_{i-1}$ does not satisfy property (SD.1). Playing from $(x, y)$ to $\left(A_{j}, B_{j}\right)$ is thus allowed. Indeed, assume on the contrary that $\left(x-A_{j}, y-B_{j}\right)$ belongs to $\mathcal{P}-\mathcal{P}$ and is of the form $\left(A_{n}-A_{m}, B_{n}-B_{m}\right)$ (the other case $\left(A_{m}-B_{n}, B_{m}-A_{n}\right)$ cannot occur). It would mean that there exists a factor $f=f_{1} \cdots f_{l}$ of $\mathbf{w}$ such that $\sum_{k=1}^{l} f_{k}=\left(i-j, B_{i}-1-B_{j}\right)$. Necessarily we have $l \leq i-j$. If $l<i-j$, then $\sum_{k=1}^{l} \pi_{2}\left(f_{k}\right) \leq \sum_{k=j}^{i-2} \pi_{2}\left(w_{k}\right)+1$ since $\pi_{2}(\mathbf{w})$ is Sturmian. But $\sum_{k=1}^{l} \pi_{2}\left(f_{k}\right)=B_{i}-1-B_{j}=\sum_{k=j}^{i-1} \pi_{2}\left(w_{k}\right)-1$, leading to the contradiction $\pi_{2}\left(w_{i-1}\right) \leq 2$. Hence $l=i-j$, meaning that $f$ is a factor of $\mathbf{w}$ over $\{a, b\}^{*}$ of length $(i-j)$. Since $w_{j} \cdots w_{i-1}$ does not satisfy property (SD.1), we get $|f|_{b} \geq\left|w_{j} \cdots w_{i-1}\right|_{b}$. In other words, we have $\sum_{k=1}^{l} \pi_{2}\left(f_{k}\right)>\sum_{k=j}^{i-1} \pi_{2}\left(w_{k}\right)-1=B_{i}-1-B_{j}$, a contradiction.

Putting together Theorems 45 and 50 yields the characterization.

Corollary 51. The set $\mathcal{P}$ is admissible if and only if the 4 -tuple $(\alpha, \beta, \gamma, \delta)$ is $C A T$.

\section{Characterizing CAT 4-tuples}

In the previous section, we have described combinatorial conditions (expressed by Definition 41 and Definition 42) on the word $\mathbf{w}_{+}$or $\mathbf{w}_{-}$leading to the existence of an admissible set. In this section, we translate these combinatorial conditions into an algebraic setting better suited to tests.

The tests described in this section are all of the following kind. Take two intervals $I, J$ over $[0,1)$ interpreted as intervals over the unit circle $\mathbb{T}^{1}=\mathbb{R} / \mathbb{Z}$, i.e., if $a>b$, then the interval $[a, b)$ is $[a, 1) \cup[0, b)$. For a given 4 -tuple $(\alpha, \beta, \gamma, \delta)$ of real numbers, we ask, whether or not there exists some $i$ such that $R_{\alpha, \beta}^{i}(\gamma, \delta) \in I \times J$. 
Definition 52. The real numbers $\alpha, \beta, 1$ are rationally independent (i.e., linearly independent over $\mathbb{Q}$ ), if whenever there exist integers $p$ and $q$ such that $p \alpha+q \beta$ is an integer, then $p=q=0$.

The extension of the density theorem of Kronecker is well-known: the set

$$
\left\{R_{\alpha, \beta}^{i}(\gamma, \delta)=(\{i \alpha+\gamma\},\{i \beta+\delta\}) \in \mathbb{T}^{2} \mid i \in \mathbb{N}\right\}
$$

is dense in $\mathbb{T}^{2}$ if and only if $\alpha, \beta, 1$ are rationally independent [19. So, in that latter case, there exist infinitely many $i$ such that $R_{\alpha, \beta}^{i}(\gamma, \delta)$ belongs to a non-empty interval $I \times J$.

If $\alpha, \beta, 1$ are rationally dependent, since $\alpha$ and $\beta$ are irrational numbers, there exist integers $p, q, r$ with $p, q \neq 0$ such that $p \alpha+q \beta=r$. From (3), we deduce that $q \beta^{2}+(p-q-r) \beta+r=0$, i.e., $\beta$ is thus an algebraic number of degree 2 . Of course, the same conclusion holds for $\alpha$. In this situation, the set of points $\left\{R_{\alpha, \beta}^{n}(\gamma, \delta) \mid n \in \mathbb{N}\right\}$ is dense on a straight line in $\mathbb{T}^{2}$ with rational slope, see for instance Example 53 . Hence the initial question is reduced to determine whether or not a line intersect a rectangle. Moreover, if $\alpha$ and $\beta$ are irrational numbers satisfying (3) but they are not algebraic of degree 2 , then $\alpha, \beta, 1$ are rationally independent.

Example 53. Consider the positive root $\alpha=(3+\sqrt{17}) / 2$ of $x^{2}-3 x-2$. We get $\beta=(7+\sqrt{17}) / 8$ and $\alpha=4 \beta-2$. Hence, we get

$$
R_{\alpha, \beta}^{n}(x, y)=(\{x+n \alpha\},\{y+n \beta\})=(\{x+4 n \beta\},\{y+n \beta\})
$$

showing that $R_{\alpha, \beta}$ corresponds to a translation of $\{\beta\}(4,1)$ in $\mathbb{T}^{2}$. Since $\beta$ is irrational, thanks to Kronecker theorem, the set of points $\left\{R_{\alpha, \beta}^{n}(x, y) \mid n \in \mathbb{N}\right\}$ is dense on the straight line in $\mathbb{T}^{2}$ with rational slope $1 / 4$ and passing through $(x, y)$.

6.1. Testing a disqualifying prefix in $\mathbf{w}_{+}$. Let $p=w_{0} u_{1} \cdots u_{n}=w_{0} u$ be the prefix of length $n+1$ of $\mathbf{w}_{+}$. If $u_{1} \cdots u_{n-1}$ is not a palindrome or if $u \notin\{a, b\}^{*}$ or if $2 B_{1} \notin\left\{\pi_{2}\left(u_{n}\right)-1, \pi_{2}\left(u_{n}\right), \pi_{2}\left(u_{n}\right)+1\right\}$, then $p$ is not disqualifying ${ }^{+}$. Otherwise, one of the following three situations may occur.

If $2 B_{1}=\pi_{2}\left(u_{n}\right)-1$, then $p$ is disqualifying ${ }^{+}$if and only if $\pi_{1}(u)$ is light, $\pi_{2}(u)$ is heavy, i.e., $R_{\alpha, \beta}(\gamma, \delta) \in I_{L, \alpha}(n) \times I_{H, \beta}(n)$ where, as in (9), $I_{L, \alpha}(n)=[0,1-\{n \alpha\})$ and $I_{H, \alpha}(n)=[1-\{n \beta\}, 1)$, and there exists a factor $v \in \mathrm{Fac}_{n}\left(\mathbf{w}_{+}\right)$such that $\pi_{1}(v)$ is heavy, $\pi_{2}(u)$ is light, i.e., there exists $i$ such that

$$
R_{\alpha, \beta}^{i}(\gamma, \delta) \in I_{H, \alpha} \times I_{L, \beta}(n) .
$$

If $2 B_{1}=\pi_{2}\left(u_{n}\right)$, then $p$ is disqualifying ${ }^{+}$if and only if $\pi_{1}(u)$ is light and there exists a factor $v \in \operatorname{Fac}_{n}\left(\mathbf{w}_{+}\right)$such that $\pi_{1}(v)$ is heavy and both $\pi_{2}(u)$ and $\pi_{2}(v)$ are either light or heavy, i.e., there exists $i$ such that

$$
\begin{aligned}
& R_{\alpha, \beta}^{i}(\gamma, \delta) \in I_{H, \alpha} \times I_{L, \beta}(n) \text { if } R_{\alpha, \beta}(\gamma, \delta) \in I_{L, \alpha}(n) \times I_{L, \beta}(n), \\
& R_{\alpha, \beta}^{i}(\gamma, \delta) \in I_{H, \alpha} \times I_{H, \beta}(n) \text { if } R_{\alpha, \beta}(\gamma, \delta) \in I_{L, \alpha}(n) \times I_{H, \beta}(n) .
\end{aligned}
$$

If $2 B_{1}=\pi_{2}\left(u_{n}\right)+1$, then $p$ is disqualifying ${ }^{+}$if and only if $\pi_{1}(u)$ and $\pi_{2}(u)$ are light, i.e., $R_{\alpha, \beta}(\gamma, \delta) \in I_{L, \alpha}(n) \times I_{L, \beta}(n)$ and there exists a factor $v \in \operatorname{Fac}_{n}\left(\mathbf{w}_{+}\right)$ such that $\pi_{1}(v)$ and $\pi_{2}(u)$ are heavy, i.e., there exists $i$ such that

$$
R_{\alpha, \beta}^{i}(\gamma, \delta) \in I_{H, \alpha} \times I_{H, \beta}(n) .
$$




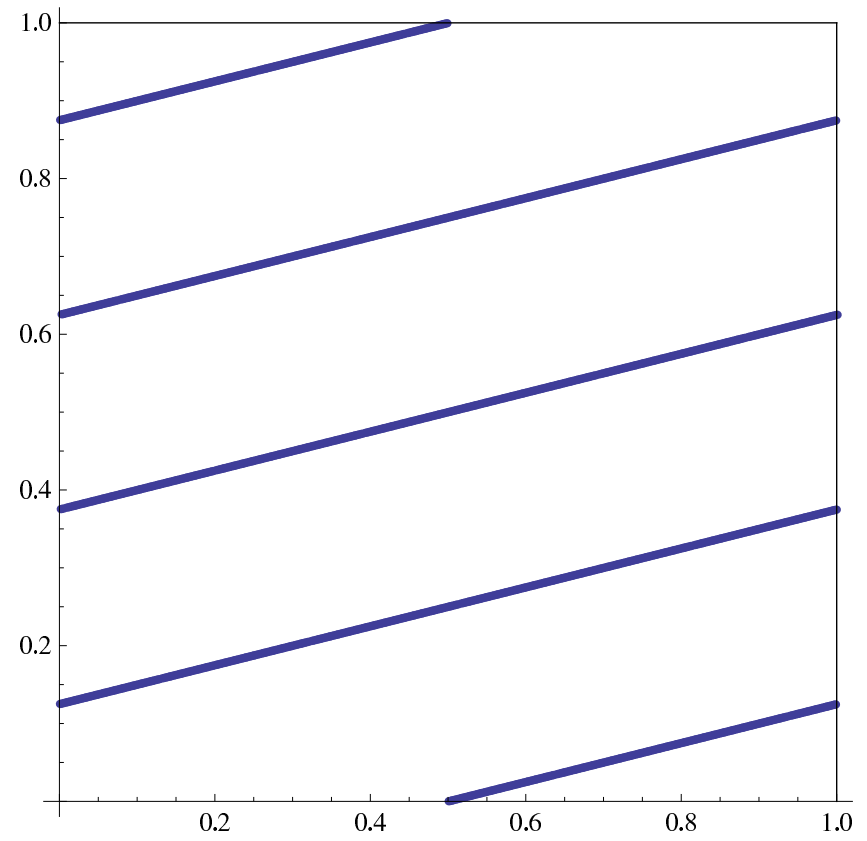

Figure 7. An example of orbit when $\alpha, \beta, 1$ are rationally dependent.

6.2. Testing (SD.1). Given $u=u_{1} \cdots u_{n} \in\{a, b\}^{*} \cap \operatorname{Fac}\left(\mathbf{w}_{-}\right)$, we can proceed as follows to decide whether or not $u$ satisfies (SD.1). If $\pi_{2}\left(u_{1} \cdots u_{n}\right)$ is light, i.e., $\left|\pi_{2}\left(u_{1} \cdots u_{n}\right)\right|_{\lfloor\beta\rfloor+1}=\lceil n\{\beta\}\rceil-1$, then $u$ does not satisfy (SD.1). Otherwise, $\pi_{2}\left(u_{1} \cdots u_{n}\right)$ is heavy. In that case, $u$ satisfies (SD.1) if and only if, there exists an integer $i$ such that

- $1^{n}$ occurs in $\mathbf{s}_{\alpha, \gamma}$ in position $i$ and,

- the factor of length $n$ occurring in $\mathbf{s}_{\beta, \delta}$ in position $i$ is light.

These last two conditions can be tested as follows. As in (8), consider the two intervals

$$
I_{1^{n}, \alpha}=I_{1} \cap R_{\alpha}^{-1}\left(I_{1}\right) \cap \cdots \cap R_{\alpha}^{-n+1}\left(I_{1}\right) \text { where } I_{1}=[0,1-\{\alpha\})
$$

and, as in (9), $I_{L, \beta}(n)=[0,1-\{n \beta\})$. Using Lemma 32, the two above conditions hold true if and only if $I_{1^{n}, \alpha} \neq \emptyset$ and there exists $i$ such that

$$
R_{\alpha, \beta}^{i}(\gamma, \delta) \in I_{1^{n}, \alpha} \times I_{L, \beta}(n) .
$$

6.3. Testing (SD.2). Given $u=u_{1} \cdots u_{n} \in\{a, b\}^{*} \cap \mathrm{Fac}\left(\mathbf{w}_{-}\right)$, we can proceed as follows to decide whether or not $u$ satisfies (SD.2). If $\pi_{2}\left(u_{1} \cdots u_{n-1}\right)$ is light, i.e., $\left|\pi_{2}\left(u_{1} \cdots u_{n-1}\right)\right|_{\lfloor\beta\rfloor+1}=\lceil(n-1)\{\beta\}\rceil-1$, then $u$ does not satisfy (SD.2). Otherwise, $\pi_{2}\left(u_{1} \cdots u_{n-1}\right)$ is heavy.

If $\lceil(n-1)\{\alpha\}\rceil>2$, then any factor of length $n-1$ in $\mathbf{s}_{\alpha, \gamma}$ contains at least two symbols 2 and $u$ does not satisfy (SD.2).

If $\lceil(n-1)\{\alpha\}\rceil=1$ (resp., if $\lceil(n-1)\{\alpha\}\rceil=2)$, then any factor of length $n-1$ in $\mathbf{s}_{\alpha, \gamma}$ with exactly one symbol 2 is heavy (resp., light). In that case, $u$ satisfies (SD.2) if and only if, there exists an integer $i$ such that 
- the factor of length $n-1$ occurring in $\mathbf{s}_{\alpha, \gamma}$ in position $i$ is heavy (resp., light),

- the factor of length $n-1$ occurring in $\mathbf{s}_{\beta, \delta}$ in position $i$ is light.

The two above conditions hold true if and only if there exists $i$ such that

$$
R_{\alpha, \beta}^{i}(\gamma, \delta) \in I_{H, \alpha}(n-1) \times I_{L, \beta}(n-1)
$$

(resp., if and only if there exists $i$ such that $R_{\alpha, \beta}^{i}(\gamma, \delta) \in I_{L, \alpha}(n-1) \times I_{L, \beta}(n-1)$ ).

\section{7. $B_{1}$-SUPERADDITIVITY IS NOT A NECESSARY CONDITION FOR INVARIANCE}

As mentioned in Section 2.3, the authors of [11] ask the question whether a pair $\left(A_{n}, B_{n}\right)_{n>0}$ of non-homogeneous complementary Beatty sequences with $A_{1}=1$ provides an admissible set if and only if the sequence $\left(B_{n}\right)_{>0}$ is $B_{1}$-superadditive. In what follows we provide counterexamples to this assumption, meaning that the two notions of a CAT 4-tuple given in Definition 44 and B1-superadditivity are not equivalent. Recall that the definition of superadditivity and $B_{1}$-superadditivity is given in Definition 18.

7.1. Counterexamples with $\gamma<0$. A sequence satisfying $\gamma<0$ is never superadditive, but some are admissible. The non-superadditivity of such sequences can be easily proved by the following Lemma.

Lemma 54. Given a pair $\left(A_{n}, B_{n}\right)_{n>0}=(\lfloor n \alpha+\gamma\rfloor,\lfloor n \beta+\delta\rfloor)_{n>0}$ of non-homogeneous complementary Beatty sequences with $\gamma<0$, there exists some integers $n, m>0$ such that $B_{m}+B_{n}>B_{m+n}$.

Proof. First note that according to (11), we have $0<\delta<1$. Since $\beta$ is irrational, there exists some integer $n>0$ such that $\{\beta n+\delta\}<\frac{\delta}{2}$. With such an $n$ we have

$$
B_{n}=\lfloor\beta n+\delta\rfloor=\beta n+\delta-\{\beta n+\delta\}>\beta n+\frac{\delta}{2} .
$$

By multiplying par 2 we obtain

$$
2 B_{n}>\beta 2 n+\delta>B_{2 n},
$$

showing the desired result.

Now, all the wanted counterexamples are those satisfying Definition 44, As an illustration, take for instance the sequence with

$$
\beta=1.99+\frac{\sqrt{5}}{2}, \quad \alpha=\frac{\beta}{\beta-1}, \quad \gamma=-0.2 \text { and } \delta=-\frac{\beta \gamma}{\alpha} .
$$

The corresponding set $\mathbb{N}^{2} \cap(\mathcal{P}-\mathcal{P})$ is represented in Figure 8 with the same color code as in Example 6 .

\begin{tabular}{c|ccccccccccccccc}
$n$ & 0 & 1 & 2 & 3 & 4 & 5 & 6 & 7 & 8 & 9 & 10 & 11 & 12 & 13 & 14 \\
\hline$A_{n+1}-A_{n}$ & 1 & 1 & 2 & 1 & 2 & 1 & 2 & 1 & 2 & 1 & 2 & 1 & 1 & 2 & 1 \\
$B_{n+1}-B_{n}$ & 3 & 3 & 3 & 3 & 3 & 4 & 3 & 3 & 3 & 3 & 3 & 3 & 3 & 3 & 4 \\
\hline $\mathbf{w}_{-}$ & $a$ & $a$ & $c$ & $a$ & $c$ & $b$ & $c$ & $a$ & $c$ & $a$ & $c$ & $a$ & $a$ & $c$ & $b$
\end{tabular}

This sequence is admissible since the 4 -tuple $(\alpha, \beta, \delta, \gamma)$ is CAT. Indeed, according to Definition 44 and since $B_{1}=3$, it suffices to show that the prefixes $a$ and $a a$ are not suffix-disqualifying. Clearly, since they do not contain any symbol $b$, they satisfy neither $(S D .1)$ nor $(S D .2)$ of Definition 42 


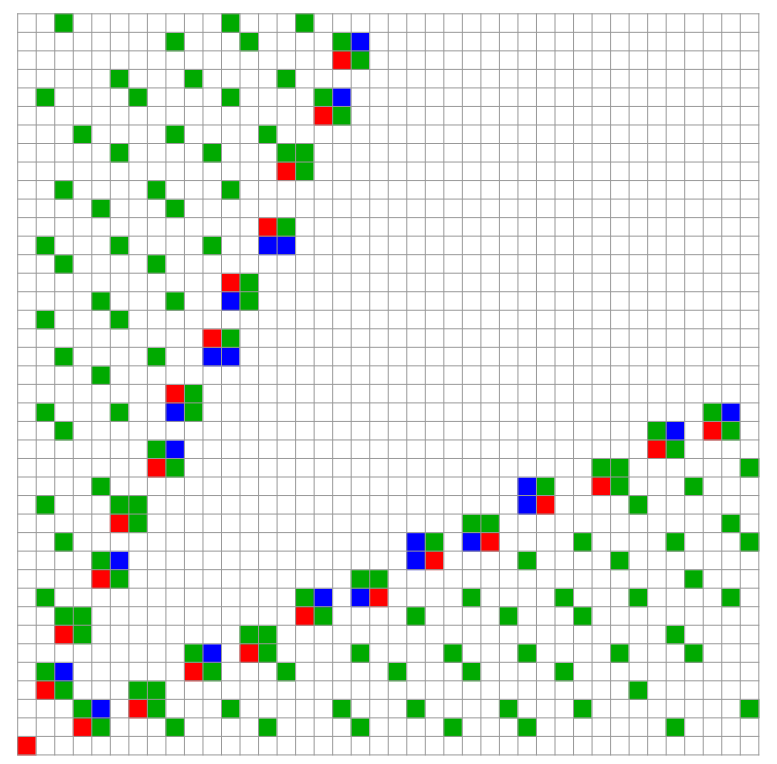

Figure 8. The set $\mathbb{N}^{2} \cap(\mathcal{P}-\mathcal{P})$ with the values in (13).

7.2. Counterexamples with $\gamma>0$. In the case where $\gamma>0$, one can also find admissible sequences that are superadditive, but not $B_{1}$-superadditive. As an example, consider the four real parameters given in Example 17. The corresponding set $\mathbb{N}^{2} \cap(\mathcal{P}-\mathcal{P})$ is represented in Figure 5 with the same color code as in Example 6 .

We have already observed in Example 19 that $\left(B_{n}\right)_{n>0}$ is not $B_{1}$-superadditive. However this sequence is superadditive as it is the case for all sequences with $\gamma>0$. It now remains to show that with the parameters given in Example 17 the sequence $\left(A_{n}, B_{n}\right)$ is admissible. For this purpose, it suffices to make use of Theorem 50 and detect directly whether or not the 4 -tuple $(\alpha, \beta, \gamma, \delta)$ is CAT. This can be easily carried on as follows.

\begin{tabular}{c|ccccccccccccccc}
$n$ & 0 & 1 & 2 & 3 & 4 & 5 & 6 & 7 & 8 & 9 & 10 & 11 & 12 & 13 & 14 \\
\hline$A_{n+1}-A_{n}$ & 1 & 1 & 2 & 1 & 1 & 1 & 1 & 1 & 1 & 1 & 2 & 1 & 1 & 1 & 1 \\
$B_{n+1}-B_{n}$ & 3 & 9 & 10 & 9 & 10 & 10 & 9 & 10 & 9 & 10 & 10 & 9 & 10 & 10 & 9 \\
\hline $\mathbf{w}_{+}$ & $e$ & $a$ & $d$ & $a$ & $b$ & $b$ & $a$ & $b$ & $a$ & $b$ & $d$ & $a$ & $b$ & $b$ & $a$
\end{tabular}

From Definition 44, since $B_{1}=3$, the only prefix of $\mathbf{w}_{+}$to test is $p=e a$. If this prefix is not disqualifying ${ }^{+}$, then the 4 -tuple $(\alpha, \beta, \gamma, \delta)$ is CAT and from Theorem 50, the sequence is admissible. In particular, $a$ is a palindrome. But $2 B_{1}=6$ and $\pi_{2}(a)=9$. Hence $2 B_{1} \notin\{8,9,10\}$ and we can conclude directly that the prefix is not disqualifying ${ }^{+}$.

\section{Concluding Remark}

The current paper does not provide any characterization of admissible sequences satisfying $B_{1}=2$. Hence the result of Larsson et al. [11 remains the best one for such sequences, asserting that $B_{1}$-superadditivity is a sufficient condition for having an admissible set. Yet, it is not a necessary condition any more in that 
context, since there also exist counterexamples of non $B_{1}$-superadditive sequences that correspond to $P$-positions of invariant games.

We have considered below two examples when $B_{1}=2$ (and $\gamma$ is negative).

Example 55. Assume $\beta=1+\sqrt{2}$ and $\delta=\sqrt{2}-1$. In this case, $\gamma \simeq-0.292$. We have

\begin{tabular}{c|cccccccccc}
$n$ & 1 & 2 & 3 & 4 & 5 & 6 & 7 & 8 & 9 & 10 \\
\hline$A_{n}$ & 1 & 3 & 4 & 6 & 8 & 9 & 11 & 13 & 15 & 16 \\
$B_{n}$ & 2 & 5 & 7 & 10 & 12 & 14 & 17 & 19 & 22 & 24
\end{tabular}

The set $\mathbb{N}^{2} \cap(\mathcal{P}-\mathcal{P})$ is represented in Figure 9 with the same color code as in Example 6. The set $\mathcal{P}$ seems to be admissible. For each blue cell, one can remove either $(1,1),(2,2)$ or $(3,3)$ to reach a red cell. The coding word is

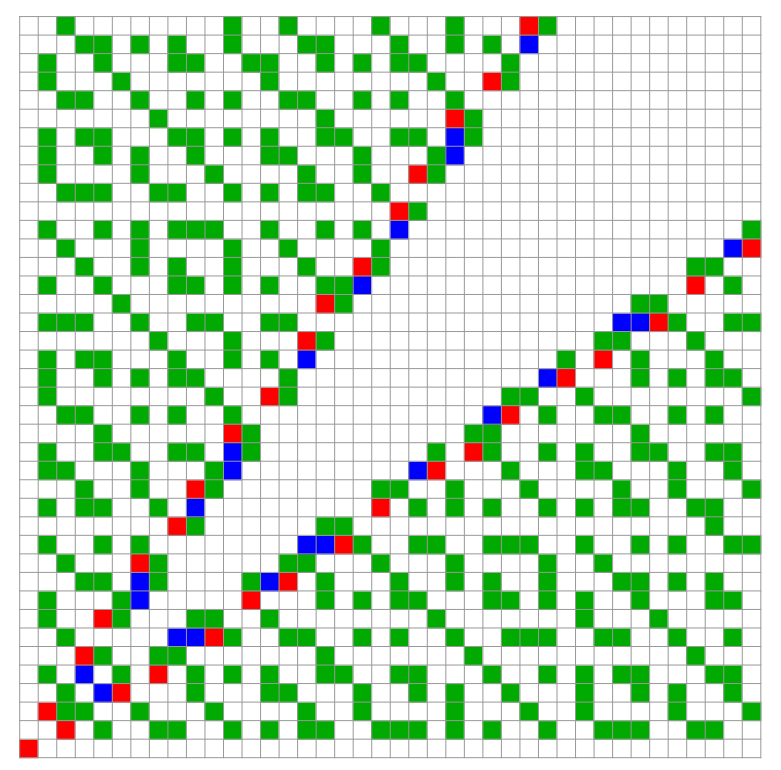

Figure 9. The set $\mathbb{N}^{2} \cap(\mathcal{P}-\mathcal{P})$ with $\beta=1+\sqrt{2}$ and $\delta=\sqrt{2}-1$.

The set $\mathcal{P}$ seems to be admissible.

$$
\mathbf{w}_{-}=\text {acdadcadcdacdadcdacdadcadcdadcad } \cdots
$$

but one should find an alternative to Definition 44. As we will see in the next example, it is not enough to have a condition on the first letter of $\mathbf{w}_{-}$.

Example 56. Assume $\beta=0.8+\sqrt{2}$ and $\delta=\sqrt{2}-1.2$. In this case, $\gamma \simeq-0.292$. We have

\begin{tabular}{c|cccccccccc}
$n$ & 1 & 2 & 3 & 4 & 5 & 6 & 7 & 8 & 9 & 10 \\
\hline$A_{n}$ & 1 & 3 & 5 & 7 & 8 & 10 & 12 & 14 & 16 & 18 \\
$B_{n}$ & 2 & 4 & 6 & 9 & 11 & 13 & 15 & 17 & 20 & 22
\end{tabular}

The set $\mathbb{N}^{2} \cap(\mathcal{P}-\mathcal{P})$ is represented in Figure 10 with the same color code as in Example 6. The set $\mathcal{P}$ is not admissible. Indeed, with the blue cell $(8,7)$ of the form $\left(B_{n}+B_{1}, A_{n+1}\right)$ with $n=3$, one has to remove either $(2,2),(4,4)$ or $(6,6)$ to reach a red cell. But these three elements belong to $\mathcal{P}-\mathcal{P}$. The coding word is 


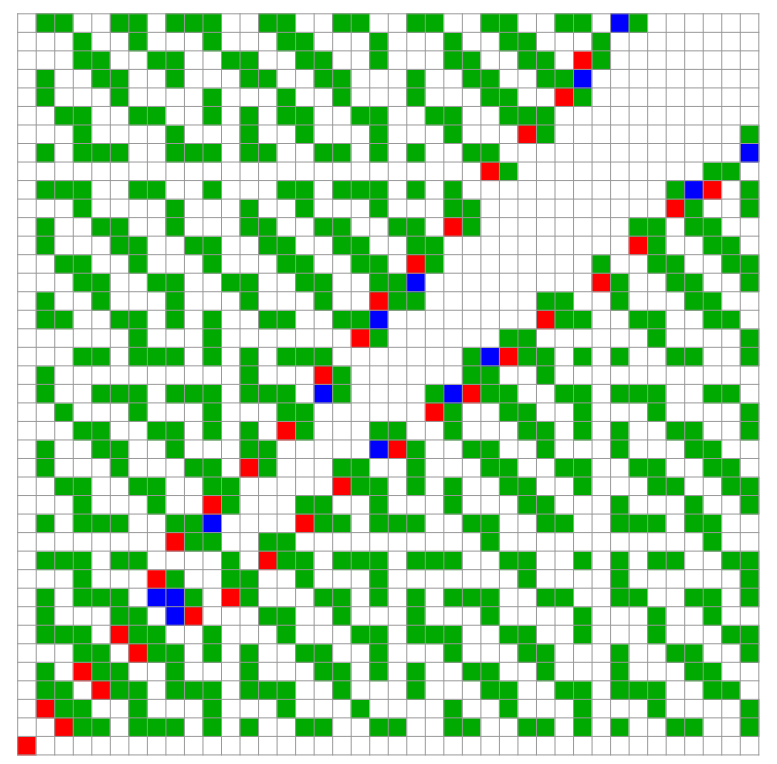

Figure 10. The set $\mathbb{N}^{2} \cap(\mathcal{P}-\mathcal{P})$ with $\beta=1+\sqrt{2}$ and $\delta=\sqrt{2}-1$;

$\mathcal{P}$ is not admissible.

$$
\mathbf{w}_{-}=\operatorname{acccdacccd} \operatorname{caccd} \operatorname{ccad} \operatorname{cccad} \operatorname{ccc} \operatorname{cocc} \boldsymbol{\cdots}
$$

but one should find an alternative to Definition 44. Indeed, as shown in the above examples, in the case $B_{1}=2$ the words $w_{+}$and $w_{-}$may have two consecutive letters in $\{c, d\}$. It makes the notions of suffix-disqualifying and disqualifying ${ }^{+}$no more relevant to define the CAT. However, one can conjecture that Definition 44 remains valid for the sequences satisfying $A_{n+2}-A_{n}<4$ for all $n$.

\section{ACKNOWLEDGMENTS}

The authors warmly thanks the referee for the important work he/she has done. His/her comments were helpful to improve the accessibility and readability of this paper.

\section{REFERENCES}

[1] V. Berthé, M. Rigo (Eds.), Combinatorics, Automata and Number Theory, Encyclopedia of Math. and its Applications 135, Cambridge University Press (2010).

[2] V. Berthé, L. Vuillon, Tilings and rotations on the torus: a two-dimensional generalization of Sturmian sequences, Discrete Math. 223 (2000), 27-53.

[3] C. L. Bouton, Nim, a game with a complete mathematical theory, Annals of Math. 3 (1905), $35-39$.

[4] E. Duchêne, A. S. Fraenkel , R. J. Nowakowski, M. Rigo, Extensions and restrictions of Wythoff's game preserving its $P$ positions, J. Comb. Theory Ser. A 117 (2010), 545-567.

[5] E. Duchêne, M. Rigo, Invariant games, Theoret. Comput. Sci. 411 (2010), 3169-3180.

[6] E. Duchêne, M. Rigo, A morphic approach to combinatorial games: The Tribonacci case, Theor. Inform. Appl. 42 (2008), 375-393.

[7] A. S. Fraenkel, The bracket function and complementary sets of integers, Canad. J. Math. 21 (1969), 6-27. 
[8] A. S. Fraenkel, How to beat your Wythoff games' opponent on three fronts, Amer. Math. Monthly 89 (1982), 353-361.

[9] A.S. Fraenkel, Complementary Iterated Floor Words and the Flora Game, SIAM J. on Discrete Math 24 (2010), 570-588.

[10] S.W. Golomb, A Mathematical Investigation of Games of "Take-Away", J. Combinatorial Theory 1 (1966), 443-458.

[11] U. Larsson, P. Hegarty, A. S. Fraenkel, Invariant and dual subtraction games resolving the Duchêne-Rigo conjecture, Theoret. Comput. Sci. 412 (2011), 729-735.

[12] U. Larsson, The $\star$-operator and invariant subtraction games, Theoret. Comput. Sci. 422 (2012), 52-58.

[13] U. Larsson, J. Wästlund, From Heaps of Matches to the Limits of Computability, Electron. J. Combin. 20 (2013), no. 3, Paper 41, 12 pp.

[14] M. Lothaire, Algebraic Combinatorics on Words, Encyclopedia of Math. and its Applications 90, Cambridge University Press (2002). its P-positions, J. Comb. Theory Ser. A 119 (2012), 1302-1314.

[15] K. O'Bryant, Fraenkel's partition and Brown's decomposition, Integers 3 (2003), A11.

[16] M. Rigo, P. Salimov, E. Vandomme, Some properties of abelian returns, J. of Integer Sequences 16 (2013) 13.2.5.

[17] P. V. Salimov, On uniform recurrence of a direct product, Disc. Math. 8 Theoret. Comput. Sci. 12 (2010), 1-8.

[18] W. A. Wythoff, A modification of the game of Nim, Nieuw Arch. Wisk. 7 (1907), 199-202.

[19] E. Zehnder, Lecutres on Dynamical Systems, Hamiltonian Vector Fields and Symplectic Capacities, EMS Textbooks in Math. (2010).

J. Cassaigne, Institut de Matématiques de Luminy, CNRS, Campus de Luminy, Case 907, 13288 Marseille Cedex 9, France

E-mail address: cassaigne@iml.univ-mrs.fr

E. Duchêne, Université de Lyon, CNRS, Université Lyon 1, LiRis, UMR5205, F-69622, FRANCE

E-mail address: eric.duchene@univ-lyon1.fr

M. Rigo, University of Liege, Department of Mathematics, Grande traverse 12 (B37), B-4000 Liège, Belgium.

E-mail address: M.Rigo@ulg.ac.be 